\title{
Nonequilibrium Stagnation-Line Radiative Heating for Fire II
}

\author{
Christopher O. Johnston ${ }^{*}$ and Brian R. Hollis ${ }^{\dagger}$ \\ NASA Langley Research Center, Hampton, VA, 23681 \\ Kenneth Sutton \\ National Institute of Aerospace, Hampton, VA, 24060
}

This paper presents a detailed analysis of the shock-layer radiative heating to the Fire II vehicle using a new air radiation model and a viscous shock-layer flowfield model. This new air radiation model contains the most up-to-date properties for modeling the atomic-line, atomic photoionization, molecular band, and non-Boltzmann processes. The applied viscous shock-layer flowfield analysis contains the same thermophysical properties and nonequilibrium models as the LAURA Navier-Stokes code. Radiation-flowfield coupling, or radiation cooling, is accounted for in detail in this study. It is shown to reduce the radiative heating by about $30 \%$ for the peak radiative heating points, while reducing the convective heating only slightly. A detailed review of past Fire II radiative heating studies is presented. It is observed that the scatter in the radiation predicted by these past studies is mostly a result of the different flowfield chemistry models and the treatment of the electronic state populations. The present predictions provide, on average throughout the trajectory, a better comparison with Fire II flight data than any previous study. The magnitude of the vacuum ultraviolet (VUV) contribution to the radiative flux is estimated from the calorimeter measurements. This is achieved using the radiometer measurements and the predicted convective heating. The VUV radiation predicted by the present model agrees well with the VUV contribution inferred from the Fire II calorimeter measurement, although only when radiation-flowfield coupling is accounted for. This agreement provides evidence that the present model accurately models the VUV radiation, which is shown to contribute significantly to the Fire II radiative heating.

\section{Nomenclature}

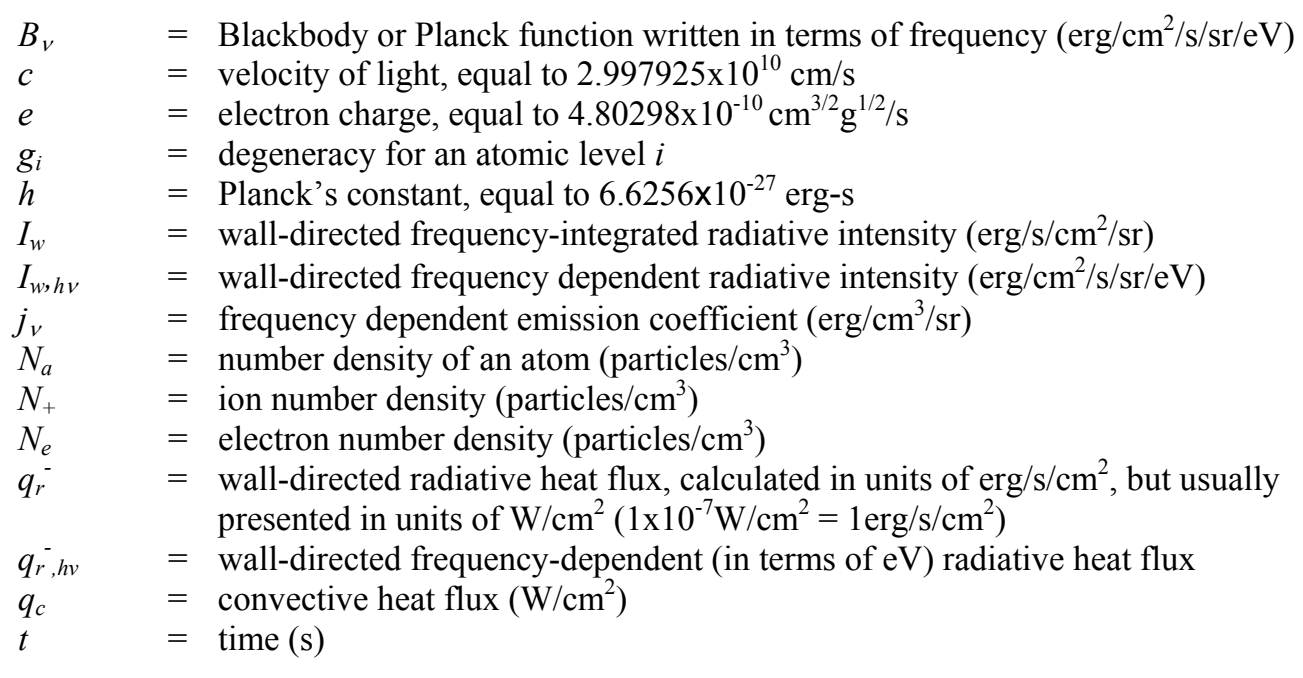

\footnotetext{
* Aerospace Engineer, Member AIAA

$\dagger$ Aerospace Engineer, Senior Member AIAA

\$Senior Research Fellow, Associate Fellow AIAA
} 


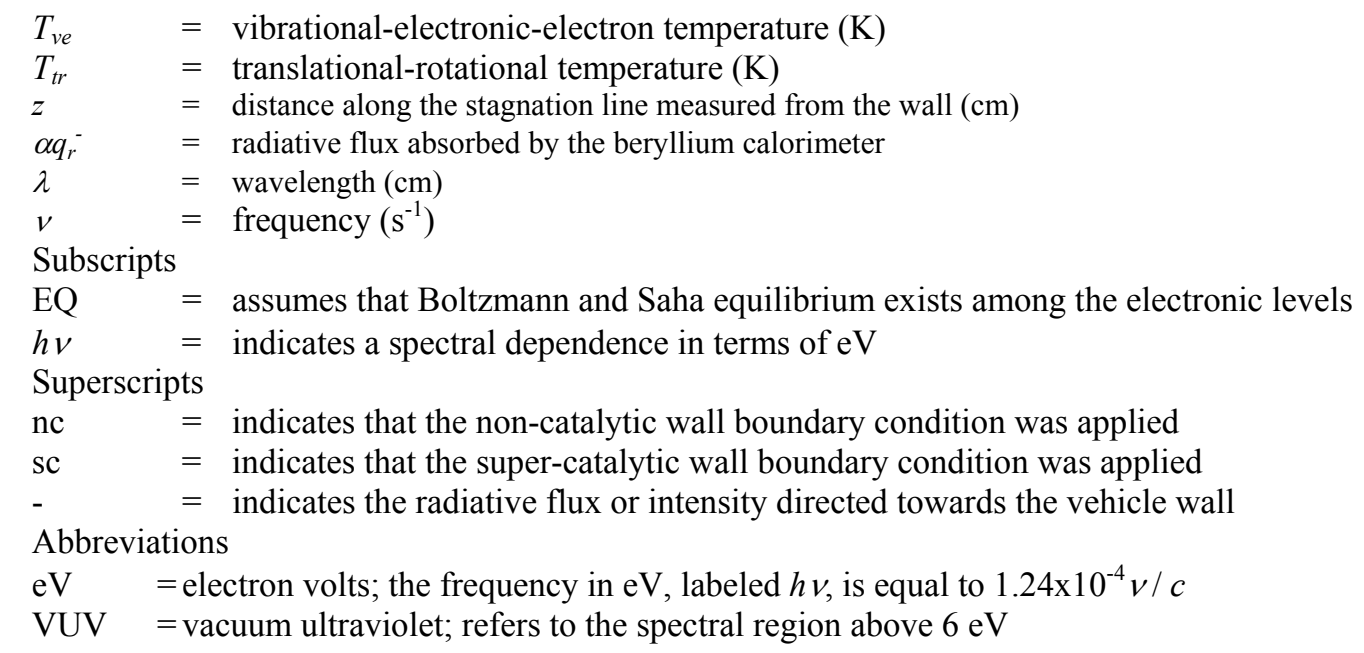

\section{Introduction}

$\mathrm{T}$ he heat-flux to a vehicle at lunar-return conditions consists of a component due to the radiative flux from the high-temperature shock-layer gas ${ }^{1}$. The prediction of this radiative flux has been studied sporadically since the beginning of manned space-flight, with significant progress being made for NASA's Apollo program. In support of this research, the Fire II flight experiment obtained radiative heating measurements at conditions similar to those for lunar-return ${ }^{2,3}$. These measurements have since become a benchmark for new radiative heating prediction algorithms. The goal of this paper is to review the past predictions of these data and to present the results of a newlydeveloped air-radiation model.

A review of past studies of the Fire II radiative heating is presented in Section II. The various predictions to the flight data are compared and the differences discussed. The flowfield and radiation model used by each researcher is defined and its influence on the comparison with the flight data is mentioned. The viscous-shock-layer flowfield model used in the present study is discussed in Section III. The number densities and temperatures from this model are compared with those from the LAURA Navier-Stokes code, and it is shown that these two flowfield models produce similar radiation predictions (when the same radiation code is applied to both). A brief description of the radiation model applied in the present study is presented in Section IV. The Fire II radiative and convective heating predicted using the present flowfield and radiation models are presented in Section V for the case where radiationflowfield coupling is not considered. These results, which are known to be physically inaccurate, are presented to provide a baseline for comparing with the radiation-flowfield coupled cases. Section VI compares the radiationflowfield coupled results of the present study with the flight data and various past predictions. The deviations of the present results from the flight data and previous studies are discussed, and the influence of non-Boltzmann radiation and flowfield coupling are assessed. The differences between the radiation predicted by a two-temperature chemical nonequilibrium VSL flowfield and a single temperature chemical equilibrium VSL flowfield are compared, which provides insight into the effect of multiple temperatures and chemical nonequilibrium on the radiative heating predictions and the effect of radiation-flowfield coupling.

\section{Review of Past Studies}

The Fire II flight experiment was flown in 1965 to obtain radiative heating data for a capsule with a scaled-down Apollo-shaped forebody ${ }^{2}$. Three non-ablating beryllium heat shields were attached to the vehicle, with the first two being jettisoned at selected points in the trajectory. Table 1 lists the flight conditions and wall temperature at various trajectory points along with the vehicle nose radius, which is different for the various heat-shields. Radiative heating data was obtained in the stagnation region with three different types of instrumentation. A total radiometer measured the radiative intensity behind a quartz window, which allowed the radiation in the spectral range of 0 to $6 \mathrm{eV}$ to be measured. A spectral radiometer provided spectrally resolved intensity data in the spectral range of 2.2 to $4.1 \mathrm{eV}$ with a resolution of about $0.04 \mathrm{eV}$ and a reported uncertainty of $\pm 23 \%$. A beryllium calorimeter measured the convective heating plus the absorbed radiative flux. The absorbed radiative flux refers to the component that is absorbed by the beryllium calorimeter over the entire spectrum. The spectral absorptance of beryllium is reported by Cornette ${ }^{3}$. 
Table 1. Trajectory points for Fire II cases

\begin{tabular}{ccccccc}
\hline$t(\mathrm{~s})$ & $\begin{array}{c}\text { Altitude } \\
(\mathrm{km})\end{array}$ & $\begin{array}{c}\text { Density } \\
\left(\mathrm{kg} / \mathrm{m}^{3}\right)\end{array}$ & $\begin{array}{c}\text { Velocity } \\
(\mathrm{km} / \mathrm{s})\end{array}$ & $\begin{array}{c}\text { Temp. } \\
(\mathrm{K})\end{array}$ & $\begin{array}{c}\text { Wall } \\
\text { Temp. }(\mathrm{K})\end{array}$ & $\begin{array}{c}\text { Physical } \\
R_{N}(\mathrm{~m})\end{array}$ \\
\hline 1634.0 & 76.42 & $3.72 \times 10^{-5}$ & 11.36 & 195 & 615 & 0.935 \\
1636.0 & 71.02 & $8.57 \times 10^{-5}$ & 11.31 & 210 & 810 & 0.935 \\
1637.5 & 67.05 & $1.47 \times 10^{-4}$ & 11.25 & 228 & 1030 & 0.935 \\
1640.5 & 59.62 & $3.86 \times 10^{-4}$ & 10.97 & 254 & 1560 & 0.935 \\
1643.0 & 53.04 & $7.80 \times 10^{-4}$ & 10.48 & 276 & 640 & 0.805 \\
1645.0 & 48.37 & $1.32 \times 10^{-3}$ & 9.83 & 285 & 1520 & 0.805 \\
1648.3 & 41.60 & $3.25 \times 10^{-3}$ & 8.10 & 267 & 503 & 0.702 \\
\hline
\end{tabular}

Many researchers have used the Fire II case as a benchmark for a new radiation code or flowfield solver ${ }^{4,5,6,7,8,9,10,11,12,13}$. Table 2 lists those studies published since 1984, which are the most relevant to modern studies. Listed along with each researcher are the flowfield equations, chemistry model, radiation code, and method of obtaining the molecular and atomic state populations implemented in the particular study. All of the studies listed here include radiation-flowfield coupling, meaning the divergence of the radiative flux is included in the energy equation.

Table 2. Summary of previous Fire II studies

\begin{tabular}{cccccc}
\hline Researcher & Ref. & $\begin{array}{c}\text { Flowfield } \\
\text { Eqs. }\end{array}$ & Chemistry $^{\#}$ & $\begin{array}{c}\text { Radiation } \\
\text { Code }\end{array}$ & $\begin{array}{c}\text { State } \\
\text { Populations }^{*}\end{array}$ \\
\hline Sutton [1984] & 4 & Euler & E & RAD/EQUIL & B \\
Balakrishnan et al. [1985] & 5 & VSL & E & RAD/EQUIL & B \\
Gupta [1987] & 6 & VSL & E & RAD/EQUIL & B \\
Bird [1987] & 7 & DSMC & NE & custom & NB \\
Carslon [1989] & 8 & VSL & E & 8-step model & NB \\
Park [1989] & 9 & VSL & NE & NEQAIR85 & NB \\
Gally [1991] & 10 & VSL & NE & RAD/EQUIL & NB \\
Greendyke et al. [1994] & 11 & NS & NE & LORAN & NB \\
Olynick et al. [1994] & 12 & NS & NE & NOVAR & B \\
Park [2004] & 13 & VSL & NE & NEQAIR04 & NB
\end{tabular}

${ }^{*}$ VSL $=$ Viscous Shock Layer, DSMC $=$ Direct Simulation Monte Carlo, NS = Navier Stokes

${ }^{\#} \mathrm{E}=$ Equilibrium, $\mathrm{NE}=$ Nonequilibrium

${ }^{\%} \mathrm{~B}=$ Boltzmann, $\mathrm{NB}=$ Non-Boltzmann

The most widely analyzed data from the three measuring devices is the total radiometer data, which was presented in Figure 13 of Cauchon ${ }^{2}$ as the radiative intensity integrated between 0 and $6 \mathrm{eV}$. Figure 1 compares the intensity values predicted by many of the researchers listed in Table 2 with the flight data. According to Cauchon ${ }^{2}$, the total radiometer data contains an uncertainty of $\pm 20 \%$, which is indicated by the error bars in the figure. The results of Olynick et al. ${ }^{12}$ compare best with the data, while the results of Gupta ${ }^{6}$ are also in good agreement throughout the trajectory. From Table 2, it is seen that Olynick et al. applied the code NOVAR, which assumed a Boltzmann distribution of electronic states, to a Navier-Stokes nonequilibrium flowfield. The NOVAR code is reported to be essentially the same as LORAN, except that it is configured for computational efficiency and assumes a Boltzmann distribution of electronic states, instead of applying the non-Boltzmann QSS model used in LORAN and NEQAIR. Note that Olynick et al.'s results compare well for the early trajectory points. This is unexpected since a large percentage of the shock layer is in nonequilibrium for these points, which should have caused the Boltzmann assumption to over-predict the radiation considerably ${ }^{11}$. In addition to the Boltzmann results, Olynick et al. also presented several cases that applied the non-Boltzmann QSS model. These results showed that the QSS and Boltzmann model agreed for the early nonequilibrium trajectory points and then diverged from each other for the later equilibrium points. This trend is inconsistent with other studies ${ }^{14}$, which show that a Boltzmann distribution of electronic states is approached in regions of chemical equilibrium.

Another interesting feature of Figure 1 is the good agreement with the data obtained by Gupta ${ }^{6}$, who applied the RAD/EQUIL code to an equilibrium VSL flowfield. In a similar study, Sutton ${ }^{4}$ also applied the RAD/EQUIL code to an equilibrium flowfield, although it was inviscid. Assuming that similar thermodynamic properties were used and that the radiation codes applied were actually the same, it would be expected that because of boundary layer 
absorption, Gupta's values should be slightly lower than Sutton's. Surprisingly, Figure 1 shows the opposite trend throughout the trajectory. Furthermore, the results of Balakrishnan et al. ${ }^{5}$ should agree very well with Gupta's, since they both applied the RAD/EQUIL code to an equilibrium VSL flowfield (although their VSL techniques were different). It is seen in Figure 1 that Gupta's and Balakrishnan et al.'s results do not compare well. Unlike Gupta's values, Balakrishnan et al.'s values are lower than Sutton's inviscid results, which indicate the expected influence of boundary layer absorption.

The most state-of-the-art studies presented in Figure 1 are those of Greendyke et al. ${ }^{11}$ and Park ${ }^{13}$, which both applied a nonequilibrium radiation model to a coupled thermochemical nonequilibrium flowfield. Although these studies agree reasonably well with the data, they do not significantly improve upon the results of older studies. A reason for this lack of improvement, especially for the early trajectory points, is the uncertainty in the nonequilibrium flowfield modeling (which was not an issue with the older chemical-equilibrium studies). This point is made clear in a study by Hartung et al. ${ }^{15}$, who adjusted some of the unknown nonequilibrium flowfield parameters and studied their effect on the radiative heating for the Fire 1631, 1634, and 1637.5 second cases. It was shown that the integrated intensity, between 0 and $6 \mathrm{eV}$, varied for the 1634 and 1637.5 cases between 1.8 to 4.4 and 11.0 to $20.0 \mathrm{~W} / \mathrm{cm}^{2}$-sr, respectively, depending upon the nonequilibrium flowfield parameters chosen. Although recommended values for the parameters are provided in the literature ${ }^{16,17,18}$, the evidence supporting these values is
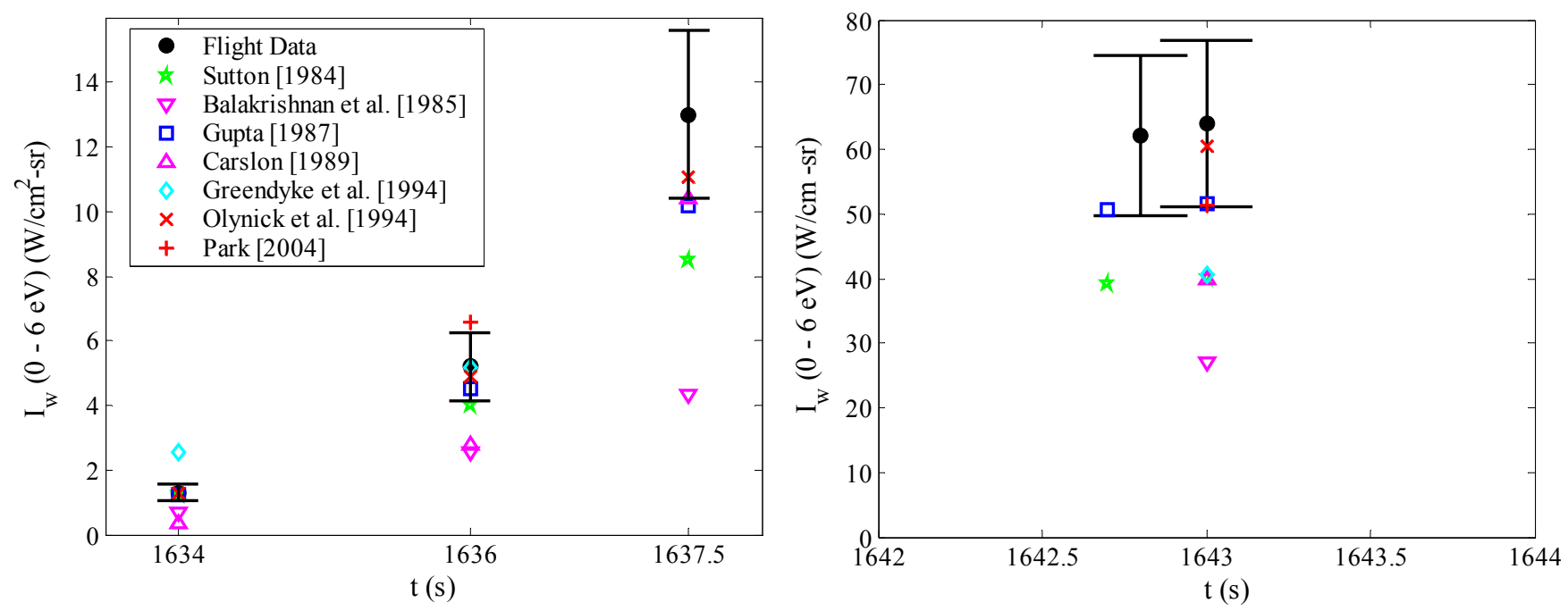

Figure 1. Comparison of past predictions for the partial intensity at the wall with the Fire II total radiometer data throughout the trajectory.

The frequency-integrated data from the spectral radiometer (between 2.2 and $4.1 \mathrm{eV}$ ), presented in Figure 13 of Cauchon $^{2}$, has been the subject of comparison by only a few researchers. The comparison between the data and predictions are shown in Figure 2. The two "Flight Data" lines represent the upper and lower limit of the data scatter. The predictions all agree reasonably well, although none of them stay within the data scatter throughout the entire trajectory. An interesting aspect of this comparison is the close agreement of the values predicted by Sutton and Balakrishnan et al., as opposed to Figure 1 where the two differ significantly, especially near the peak heating point. A few studies also compared their predictions with the spectrally-resolved data ${ }^{19}$ from the spectral radiometer $^{13,15}$. Park ${ }^{13}$ showed that his predicted spectrum compared well with the measured spectrum for the $1643 \mathrm{~s}$ point. This result is consistent with the decent comparison of the frequency-integrated values presented in Figure 2. Hartung et al. ${ }^{15}$ compared their computed spectra obtained with the LORAN code, with the data for the 1631,1634 , and $1637.5 \mathrm{~s}$ trajectory points. The agreement with the data for these nonequilibrium cases was not very good, but since they did not present values for the integrated intensity between 2.2 and $4.1 \mathrm{eV}$, it is difficult to quantify the disagreement. From Figure 5 of Hartung ${ }^{20}$ and Figures 6 and 7 presented by Greendyke ${ }^{21}$, it is suspected that the non-Boltzmann modeling of $\mathrm{N}_{2}$ and $\mathrm{N}_{2}{ }^{+}$are likely responsible for this poor agreement with the data. The 2.2 to 4.1 $\mathrm{eV}$ region of the spectrum is dominated by the $\mathrm{N}_{2}\left(1^{+}\right)$and $\mathrm{N}_{2}^{+}\left(1^{-}\right)$bands, which emit strongly in the nonequilibrium region near the shock. The LORAN code applied by Hartung uses the same non-Boltzmann QSS model as NEQAIR.

The Fire II calorimeter data presented by Cornette ${ }^{3}$ provide heating values that contain the convective heating plus the contribution of the radiative flux absorbed by the beryllium calorimeter. A comparison of the calorimeter 
data with various predictions, throughout the trajectory, is presented in Figure 3 . The $\pm 5 \%$ error bars on the data in this figure indicate the measurement uncertainty according to Cornette ${ }^{3}$. Although the flight data cannot be separated into convective and radiative components, Figure 4 compares the calculated values of these components, whose values add together to produce the values shown in Figure 3. The best comparisons with the data in Figure 3 are the results of Olynick et al. ${ }^{12}$, as was the case for the radiometer data in Figure 1. It is also seen in Figure 3 that the results of Sutton ${ }^{4}$ and Gupta ${ }^{6}$ are in close agreement with each other throughout the trajectory. This agreement is due to their offsetting differences in the radiative and convective components. Surprisingly, Sutton's radiative component is larger than Gupta's, which is the opposite of what was seen in Figure 1 for the 0 to $6 \mathrm{eV}$ integrated intensity. This indicates that Sutton predicted a larger radiative contribution from the spectral region above $6 \mathrm{eV}$ (the vacuum ultraviolet) than Gupta. This difference is likely due to boundary layer absorption, which influences the vacuum ultraviolet more than any other spectral region. The results of Greendyke et al. ${ }^{11}$ provide the worst comparison with the data. It is seen that both the convective and radiative predictions are too low, especially near peak heating. Note that the large difference in convective heating predicted by Greendyke et al. and Olynick et al. is present even though both studies applied a nonequilibrium Navier-Stokes flowfield with a super-catalytic wall boundary condition.

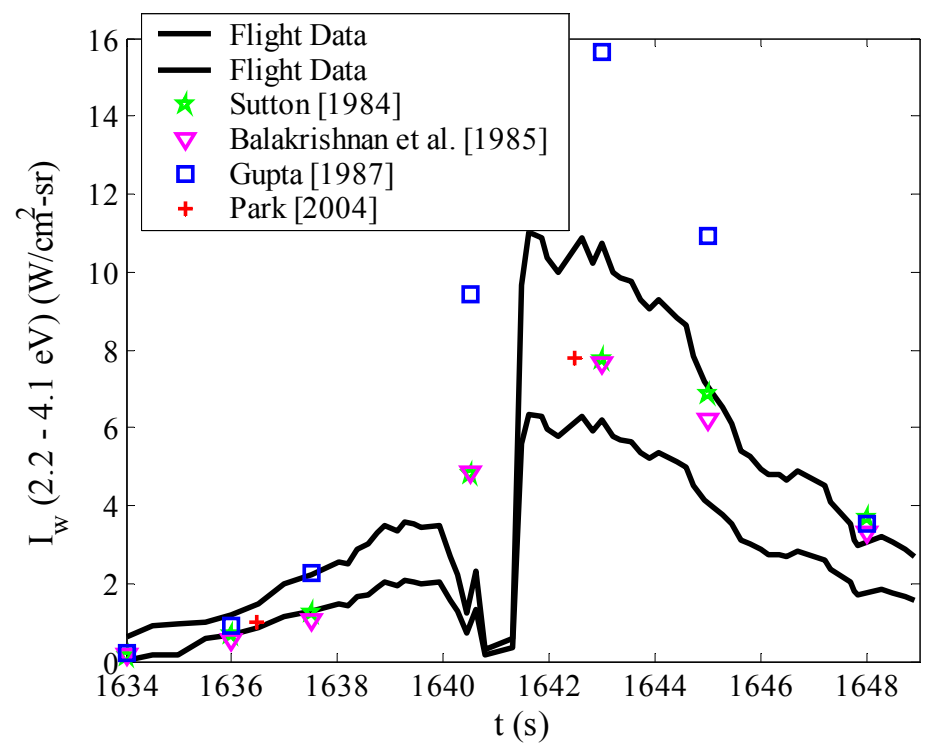

Figure 2. Comparison of past predictions with the frequency-integrated Fire II spectral radiometer data.

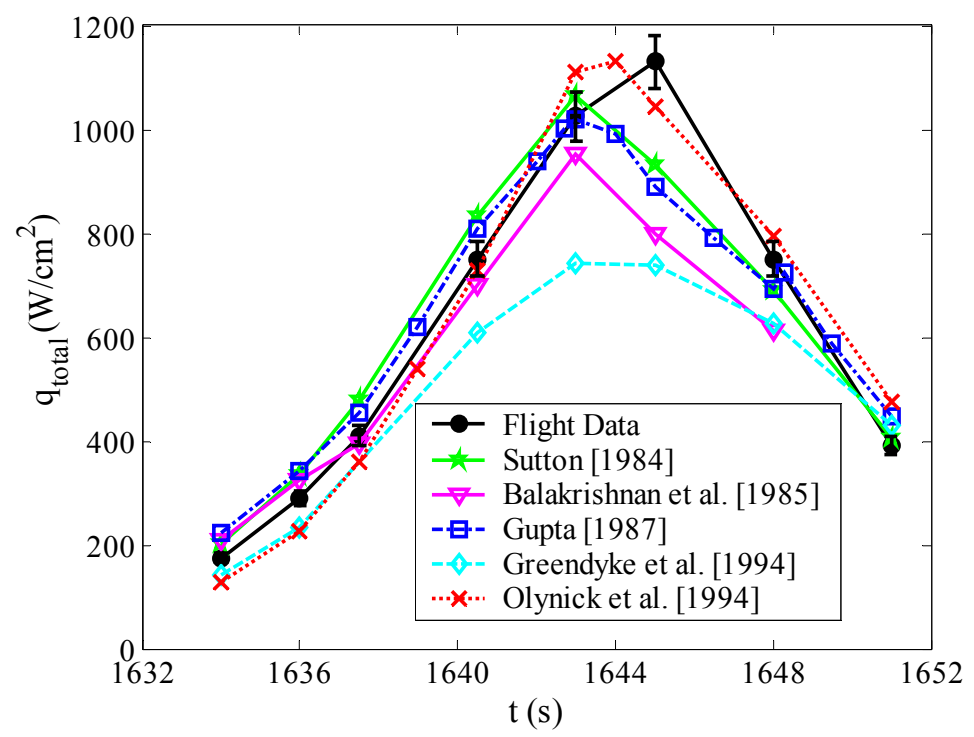

Figure 3. Comparison of past predictions for the total absorbed heat flux with the Fire II calorimeter data.

American Institute of Aeronautics and Astronautics 

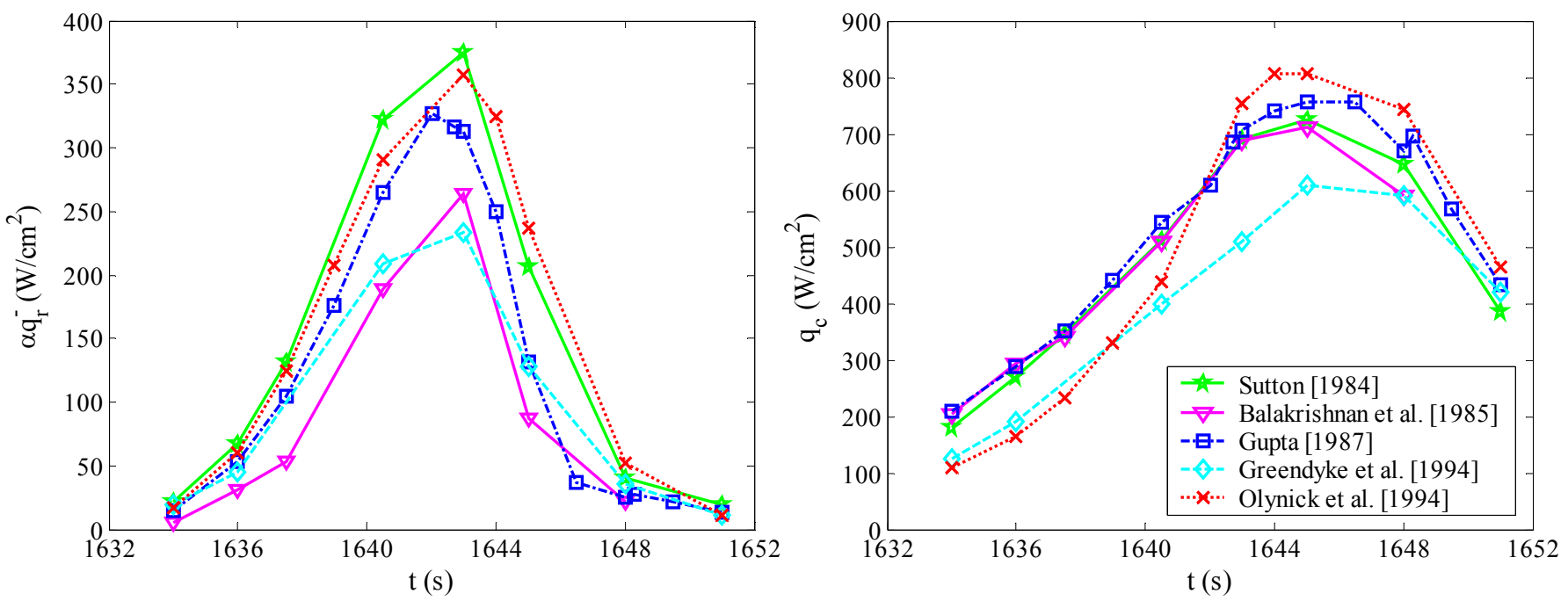

Figure 4. Comparisons of the predictions by various researchers for the radiation absorbed by the calorimeter $\left(\alpha q_{r}^{-}\right)$and the convective heating $\left(q_{c}\right)$.

\section{Flowfield Model}

The flowfield and radiation model applied in the present study is discussed in detail by Johnston ${ }^{22}$. A viscous shock layer (VSL) method ${ }^{23}$ was applied for the flowfield model. This method allows for a computationally efficient solution that retains all of the important flowfield physics for the stagnation region of a blunt body. Only the stagnation line flowfield was considered in the present study, although the same method may be used to obtain solutions downstream of the stagnation line. The same kinetic models and thermophysical properties are applied in the present VSL code as are applied in the LAURA code ${ }^{24}$. The air model applied consists of the following 11species: $\mathrm{N}_{2}, \mathrm{~N}_{2}{ }^{+}, \mathrm{O}_{2}, \mathrm{O}_{2}{ }^{+}, \mathrm{NO}, \mathrm{NO}^{+}, \mathrm{N}, \mathrm{N}^{+}, \mathrm{O}, \mathrm{O}^{+}$, and $\mathrm{e}^{-}$. The forward chemical rates were taken from Park ${ }^{25}$, while the backward rates were obtained by applying detailed balancing; with the equilibrium constants calculated using curve-fits from McBride et $\mathrm{al}^{26}$. These curve-fits were also used to calculate the specific heat and enthalpy values for each species. The collision cross-sections required for the calculation of the diffusion, viscosity, and thermal conductivity coefficients were taken from Gupta et al. ${ }^{27}$. Multicomponent diffusion was modeled using the "approximate-corrected" approach presented by Sutton and Gnoffo ${ }^{28}$.

To validate the developed VSL model for lunar return conditions, comparisons were made with Navier-Stokes results produced by the LAURA code for Fire II cases. The temperature and species number densities (relevant to the radiation calculation) predicted by the VSL method and the LAURA code are compared in Figure 5 for the Fire II $1636 \mathrm{~s}$ trajectory point. This point was chosen because the shock-layer contains both a region of chemical equilibrium and nonequilibrium. In these figures, the vehicle wall is located at $z=0$, where $z$ is the distance along the stagnation line. The consequence of the different treatments of the bow shock wave by the two methods is clearly shown in these figures. The "discrete shock" model of the VSL approach is seen to result in a larger translational-rotational temperature directly behind the shock, even with the application of the shock-slip equations. Along with beginning at a higher temperature directly behind the shock, the $T_{t r}$ values in Figure 5 are seen to behave slightly different in the nonequilibrium region directly behind the shock. This is another consequence of the discrete shock treatment, which results from $T_{t r}$ beginning its relaxation process behind the shock at a higher temperature than for the LAURA case. The $T_{v e}$ values, on the other hand, are in relatively good agreement in the nonequilibrium regions. This is fortunate because $T_{v e}$ governs the radiation. Other than the differences near the shock, the temperature profiles shown in Figure 5 closely agree throughout the rest of the shock-layer. The locations at which the two temperatures equilibrate compare well between the methods, as do the values of the temperatures in the equilibrium regions of the layer, which agree within $1 \%$ for this case. This close agreement in the equilibrium region of the shock-layer is required for the prediction of similar radiative emission values, because of the exponential $T_{v e}$ dependence of the radiative emission. This exponential dependence is due to the Boltzmann distribution of the upper electronic state of the various radiative transitions, and hence is only true in the regions of chemical equilibrium where a Boltzmann distribution is approached. Conversely to this exponential dependence on temperature, most of 
the radiative emission depends linearly on the species number densities (the atomic bound-free emission has essentially a quadratic dependence). The disagreement in the equilibrium regions of no more than $5 \%$ for the present model is therefore regarded as sufficient.
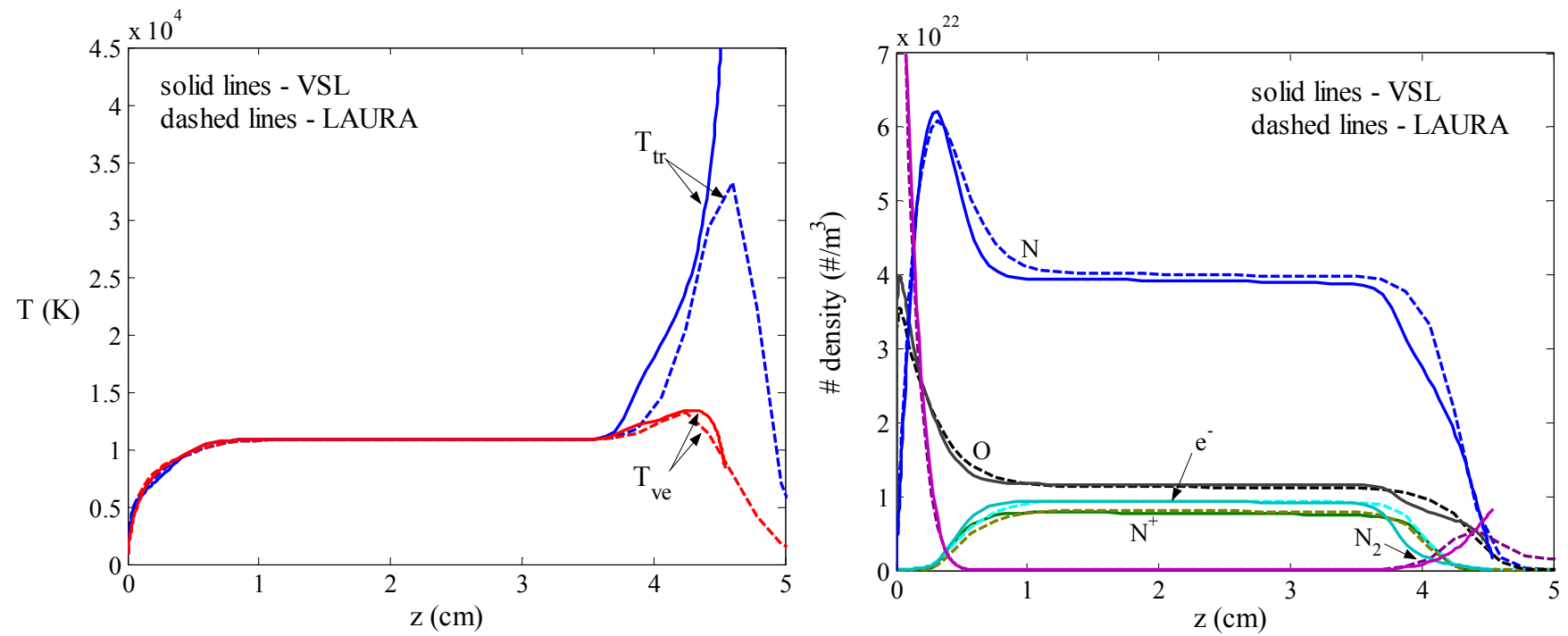

Figure 5. Comparison of the VSL and LAURA stagnation line temperature profiles and number densities for the Fire 1636 case.

To make certain that the VSL method provides a sufficient flowfield model for predicting radiative heating, the radiation model to be discussed in the next section was applied to the Fire II 1634 and 1636 s flowfields. The radiation model for these calculations includes the non-Boltzmann model for the atomic and molecular electronic states. Figure 6 presents the wall-directed radiative flux for these cases resulting from the uncoupled VSL and LAURA flowfields. The values at the wall $(z=0)$ agree within $5 \%$ for the $1636 \mathrm{~s}$ case and $1 \%$ for the $1634 \mathrm{~s}$ case, while the values throughout the shock-layer agree closely as well. This agreement throughout the shock layer is required for the accurate modeling of radiation-flowfield coupling, which although not included here, is dependent on the wall-directed (and shock-directed) radiative flux at each point through the shock-layer, as will be discussed in Section VI.

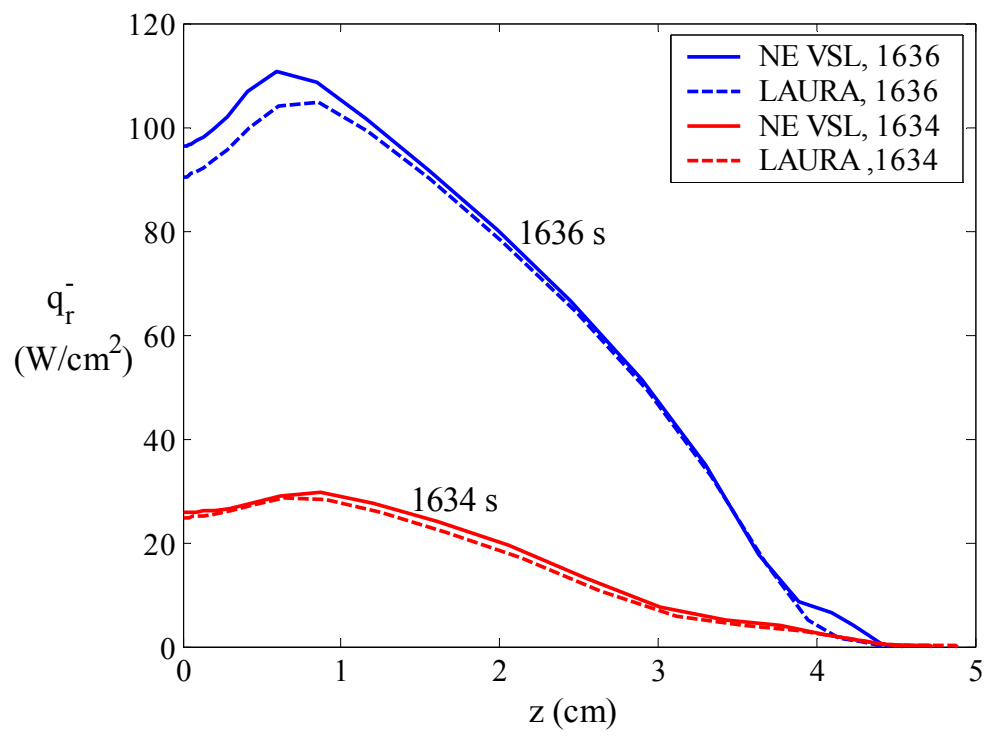

Figure 6. Wall directed radiative heat flux values predicted by the non-Boltzmann radiation models for the various flowfields. 


\section{Radiation Model}

The radiation model applied in the present study is discussed in detail by Johnston ${ }^{22}$. This model is based on a set of atomic levels and lines obtained from the National Institute of Standards and Technology (NIST) online database $^{29}$ and atomic bound-free cross sections from the Opacity project's online TOPbase ${ }^{30}$. The molecular band systems are treated using a smeared-rotational band (SRB) model ${ }^{31}$. The molecular data for modeling these band systems are obtained from Laux ${ }^{32}$, except for the vacuum ultraviolet $\mathrm{N}_{2}$ systems, which are obtained from various other sources $^{33,34,35}$. The non-Boltzmann modeling of the atomic and molecular electronic states is based on a set of electron-impact excitation rates compiled from the literature and presented in detail by Johnston ${ }^{22}$. Following the work of Park ${ }^{36}$, the quasi-steady state assumption is made when solving the Master Equation. The tangent-slab approximation is applied to calculate radiative flux and the divergence of the radiative flux, which is required for the radiation-flowfield coupling procedure.

\section{Uncoupled Radiative Heating for Fire II}

The Fire II flight experiment was discussed in detail in Section II. Along with presenting the flight data, it was shown in this section that there is a large scatter among the previous theoretical predictions of this data. Furthermore, no prediction method compared well throughout both the early nonequilibrium region of the trajectory and in the later mostly equilibrium peak-heating region. It was shown that the more recent and sophisticated prediction methods did not lead to a consistently better comparison with the data relative to older prediction methods. The goal of this section and Section VI is to compare the results of the new flowfield and radiation models presented in Sections III and IV with the flight data and previous predictions. It will be shown that the present model provides a better comparison with the data, on average over the trajectory, than any previous study. Although the uncoupled results are known to be physically inaccurate, they are presented in this section so that they may be compared with the uncoupled values predicted by previous studies. This allows the differences in the flowfield and radiation models to be examined without the complication of radiation-flowfield coupling. Furthermore, it will be insightful to compare these uncoupled values with the coupled values presented in the next section.

The chemical kinetics and thermophysical properties applied in the present flowfield model were discussed in Section III. Examples of uncoupled Fire II flowfields were also presented in that section, and shown to provide uncoupled radiative heating values consistent with those resulting from a LAURA (Navier-Stokes) flowfield. Table 3 lists the various radiative components predicted with the present uncoupled nonequilibrium VSL model and radiation model discussed in Sections III and IV. The $q_{r}^{-}$and $I_{w}$ values listed here represent the wall-directed radiative flux and wall-directed intensity, respectively, at the wall $(z=0)$. The numbers in parenthesis in the header column represent the spectral range, in $\mathrm{eV}$, included in each value. The $\alpha q_{r}{ }^{-}$values represent the radiation absorbed by the beryllium calorimeter, which is a quantity required for comparison with the calorimeter data (as mentioned previously in the discussion of Figure 4). Also required for the comparison with the calorimeter data is the convective heating $\left(q_{c}\right)$, which is listed in this table assuming both a super-catalytic (sc) and non-catalytic (nc) wall. The difference in the radiation predicted by these two catalyticity assumptions is negligible, and so only the results from the super-catalytic case are presented here.

Table 3. Uncoupled convective and radiative heat flux values $\left(\mathrm{W} / \mathrm{cm}^{2}\right)$ and radiative intensities $\left(\mathrm{W} / \mathrm{cm}^{2}\right.$-sr) for the Fire II vehicle obtained using the NE VSL flowfield model.

\begin{tabular}{ccc|ccc|ccc|ccc}
\hline$t(\mathrm{~s})$ & $\boldsymbol{q}_{\boldsymbol{c}}$ & $\boldsymbol{q}_{\boldsymbol{c}}$ & $\alpha \boldsymbol{q}_{\boldsymbol{r}}^{-}$ & $\alpha q_{r}^{-}$ & $\alpha q_{r}^{-}$ & $\boldsymbol{q}_{\boldsymbol{r}}^{-}$ & $q_{r}^{-}$ & $q_{r}^{-}$ & $\boldsymbol{I}_{\boldsymbol{w}}$ & $I_{w}$ & $I_{w}$ \\
& $\boldsymbol{s c}$ & $\boldsymbol{n c}$ & $(\mathbf{0 - 1 8})$ & $(0-6)$ & $(6-18)$ & $\mathbf{( 0 - 1 8 )}$ & $(0-6)$ & $(6-18)$ & $(\mathbf{0 - 1 8})$ & $(0-6)$ & $(6-18)$ \\
\hline 1634.0 & $\mathbf{2 2 1}$ & $\mathbf{1 1 5}$ & $\mathbf{1 9 . 2}$ & 5.53 & 13.7 & $\mathbf{2 5 . 7}$ & 10.9 & 14.8 & $\mathbf{4 . 9}$ & 1.8 & 3.2 \\
1636.0 & $\mathbf{3 1 6}$ & $\mathbf{1 9 5}$ & $\mathbf{7 1 . 6}$ & 18.7 & 52.9 & $\mathbf{9 6 . 1}$ & 38.5 & 57.7 & $\mathbf{1 9 . 5}$ & 6.4 & 13.0 \\
1637.5 & $\mathbf{3 9 8}$ & $\mathbf{2 6 6}$ & $\mathbf{1 5 2}$ & 40.8 & 111 & $\mathbf{2 0 6 . 3}$ & 85.1 & 121.2 & $\mathbf{4 2 . 6}$ & 14.5 & 28.1 \\
1640.5 & $\mathbf{5 7 8}$ & $\mathbf{4 3 9}$ & $\mathbf{4 2 7}$ & 140 & 287 & $\mathbf{6 1 5 . 5}$ & 297.0 & 318.5 & $\mathbf{1 2 9 . 6}$ & 51.8 & 77.8 \\
\hline 1643.0 & $\mathbf{7 7 7}$ & $\mathbf{6 8 9}$ & $\mathbf{5 0 0}$ & 197 & 303 & $\mathbf{7 5 5 . 5}$ & 415.8 & 339.7 & $\mathbf{1 5 6 . 2}$ & 71.9 & 84.4 \\
1645.0 & $\mathbf{8 3 5}$ & $\mathbf{7 4 3}$ & $\mathbf{2 5 3}$ & 124 & 129 & $\mathbf{4 0 3 . 7}$ & 256.5 & 147.2 & $\mathbf{7 9 . 1}$ & 42.9 & 36.3 \\
\hline 1648.3 & $\mathbf{7 5 6}$ & $\mathbf{6 7 0}$ & $\mathbf{2 5 . 6}$ & 13.2 & 12.4 & $\mathbf{4 1 . 8}$ & 25.2 & 16.6 & $\mathbf{7 . 1}$ & 4.0 & 3.1 \\
\hline
\end{tabular}

The two previous studies that present uncoupled results over the majority of the trajectory are the studies by Olynick et al. ${ }^{12}$ and Greendyke et al. ${ }^{11}$. The comparison between the $q_{r}{ }^{-}$and $I_{w}(0-6)$ values presented in Table 3 and those presented in Table $\mathrm{V}$ of Olynick et al. indicates agreement within $10 \%$ over most of the trajectory. The values presented by Greendyke et al. in their Figure 4 and 5, on the other hand, are lower than the present values by up to $30 \%$. The fact that Olynick et al.'s values agree well, while Greendyke et al.'s do not, is interesting considering that 
Olynick et al. applied a Boltzmann model and Greendyke et al. applied a non-Boltzmann model for the electronic state populations. As mentioned previously, the present study also applies a non-Boltzmann model, which is known to result in less radiation than the Boltzmann model, especially in regions of significant chemical nonequilibrium. Note that the flowfields predicted by Greendyke et al. and Olynick et al. were significantly different (see Figure 3 of Olynick et al. ${ }^{12}$ ), even though both were nonequilibrium Navier-Stokes solutions.

After the completion of the present research, the study by Hash et al.$^{37}$ of the uncoupled convective and radiative heating for Fire II became available to the authors. This work compared the convective heating predicted by various state-of-the-art Navier-Stokes codes. Because the present flowfield model applies the same energy equation and thermophysical modeling as the LAURA code, the LAURA results presented by Hash et al. should compare well with the present results. The super-catalytic and non-catalytic convective heating predicted by the LAURA code at $1636 \mathrm{~s}$ agree with the present results within $2 \%$. For the $1643 \mathrm{~s}$ case, the super-catalytic value agrees within $3 \%$ while the non-catalytic value agrees within $9 \%$. The current radiation results are $26 \%$ greater, in the $0-6 \mathrm{eV}$ range, and $47 \%$ greater, in the $6-18 \mathrm{eV}$ range, than the NEQAIR results presented by Hash et al. for the $1636 \mathrm{~s}$ case.

\section{Coupled Radiative Heating for Fire II}

The radiation components predicted by the present radiation-coupled flowfield model are presented in Table 4 for the Fire II case. The column definitions are identical to those of Table 3. To illustrate the differences between these values and the uncoupled results discussed previously, Figure 7 compares $q_{c}, q_{r}{ }^{-}$, and $\alpha q_{r}{ }^{-}$between the coupled and uncoupled cases. It is seen that the $q_{r}^{-}$and $\alpha q_{r}{ }^{-}$values are reduced by $20-30 \%$ throughout the main heating portion of the trajectory. This reduction is slightly larger than the $15-25 \%$ reduction reported by Olynick et al. ${ }^{12}$ and the $5-18 \%$ reduction reported by Greendyke et al. ${ }^{11}$. The smaller reduction found by Greendyke et al. is most likely a result of their significantly smaller uncoupled radiation prediction, which reduces the coupling effect. The discrepancy with Olynick et al.'s result is likely a result of differences in the flowfield modeling. A significant difference between Olynick et al.'s flowfield model and the present model is that Olynick et al. obtains the vibrational-electronic-electron temperature by solving the vibrational energy equation, and not the vibrationalelectronic-electron energy equation, as is done in the present model. The consequence of this treatment by Olynick et al. is that the divergence of the radiative flux does not appear in the vibrational energy equation, even though the temperature obtained from this equation governs the magnitude of the radiation. The influence of radiation on the behavior of the two temperatures is therefore fundamentally different for this model because radiation does not directly influence the vibrational-electronic-electron temperature. For the high-temperature shock-layers of present interest, the vibrational energy is very small throughout most of the shock-layer because the majority of the molecules are dissociated. Furthermore, the coupling of the vibrational energy mode to the translational energy mode is represented through vibrational-translational energy relaxation, which is shown in Eq. (55) of Gnoffo et al. ${ }^{24}$ to be proportional to the mass fractions of the molecules. For the present highly-dissociated conditions, this term is small, which results in the vibrational energy mode being weakly coupled to the translational energy mode. This indicates the possible inadequacy in solving the vibrational energy equation instead of the vibrational-electronicelectron energy equation for the high-temperature shock-layers of present interest.

Table 4. Coupled convective and radiative heat flux values $\left(\mathrm{W} / \mathrm{cm}^{2}\right)$ and radiative intensities $\left(\mathrm{W} / \mathrm{cm}^{2}\right.$-sr) for the Fire II vehicle obtained using the NE VSL flowfield model.

\begin{tabular}{ccc|ccc|ccc|ccc}
\hline$t(\mathrm{~s})$ & $\boldsymbol{q}_{\boldsymbol{c}}$ & $\boldsymbol{q}_{\boldsymbol{c}}$ & $\alpha \boldsymbol{q}_{r}^{-}$ & $\alpha q_{r}^{-}$ & $\alpha q_{r}^{-}$ & $\boldsymbol{q}_{r}^{-}$ & $q_{r}^{-}$ & $q_{r}^{-}$ & $\boldsymbol{I}_{\boldsymbol{w}}$ & $I_{w}$ & $I_{w}$ \\
& $\boldsymbol{s c}$ & $\boldsymbol{n c}$ & $(\mathbf{0 - 1 8})$ & $(0-6)$ & $(6-18)$ & $\mathbf{( 0 - 1 8 )}$ & $(0-6)$ & $(6-18)$ & $(\mathbf{0 - 1 8 )}$ & $(0-6)$ & $(6-18)$ \\
\hline 1634.0 & $\mathbf{2 1 7}$ & $\mathbf{1 1 3}$ & $\mathbf{1 7 . 2}$ & 5.3 & 11.9 & $\mathbf{2 3 . 2}$ & 10.3 & 12.9 & $\mathbf{4 . 4}$ & 1.7 & 2.8 \\
1636.0 & $\mathbf{3 0 4}$ & $\mathbf{1 8 7}$ & $\mathbf{5 9 . 4}$ & 16.7 & 42.7 & $\mathbf{8 0 . 7}$ & 34.2 & 46.5 & $\mathbf{1 6 . 2}$ & 5.7 & 10.5 \\
1637.5 & $\mathbf{3 7 7}$ & $\mathbf{2 5 3}$ & $\mathbf{1 1 9}$ & 35.0 & 83.9 & $\mathbf{1 6 5}$ & 72.6 & 92.0 & $\mathbf{3 3 . 6}$ & 12.3 & 21.3 \\
1640.5 & $\mathbf{5 3 5}$ & $\mathbf{4 1 0}$ & $\mathbf{3 0 9}$ & 112 & 196 & $\mathbf{4 5 5}$ & 236 & 219 & $\mathbf{9 4 . 2}$ & 40.8 & 53.4 \\
\hline 1643.0 & $\mathbf{7 3 3}$ & $\mathbf{6 5 4}$ & $\mathbf{3 7 0}$ & 159 & 211 & $\mathbf{5 6 7}$ & 334 & 233 & $\mathbf{1 1 5}$ & 57.3 & 57.8 \\
1645.0 & $\mathbf{8 0 7}$ & $\mathbf{7 2 1}$ & $\mathbf{2 0 8}$ & 107 & 101 & $\mathbf{3 3 8}$ & 223 & 115 & $\mathbf{6 5 . 2}$ & 37.0 & 28.3 \\
\hline 1648.3 & $\mathbf{7 5 2}$ & $\mathbf{6 7 2}$ & $\mathbf{2 5 . 6}$ & 13.2 & 12.4 & $\mathbf{4 1 . 7}$ & 25.1 & 16.6 & $\mathbf{7 . 1}$ & 4.0 & 3.1 \\
\hline
\end{tabular}

The influence of radiation-flowfield coupling on a two-temperature chemical nonequilibrium flowfield should be similar to that predicted by a single-temperature chemical equilibrium model, assuming that the electrontranslational energy exchange term is properly treated (for the two-temperature model). By comparing the present two-temperature chemical nonequilibrium VSL flowfield (NE VSL) with a chemical equilibrium VSL flowfield (E VSL), the influence of coupling on the two models may be compared. Figure 8 (a) compares the partial intensity $(0-$ 
$6 \mathrm{eV}$ ) at the wall predicted by the uncoupled and coupled NE VSL and E VSL predictions. The decrease in intensity with the addition of coupling is seen to be very similar for both flowfield models. This confirms the previous statement regarding the similarity of the coupling effect for the two flowfield models. Figure 8(b) presents the coupled and uncoupled temperature profiles for the NE VSL and E VSL models at the $1643 \mathrm{~s}$ condition. Note that the vertical-axis of this figure ranges from $10,000 \mathrm{~K}$ to $12,000 \mathrm{~K}$, for clarity. Except for the differences in the boundary layer, which is a result of the different diffusion modeling and possible nonequilibrium effects, the NE VSL and E VSL models compare very well for both the coupled and uncoupled cases. The slight separation seen between the models throughout the layer is only 10 to $20 \mathrm{~K}$, which is negligible relative to the temperatures of roughly $11,000 \mathrm{~K}$.

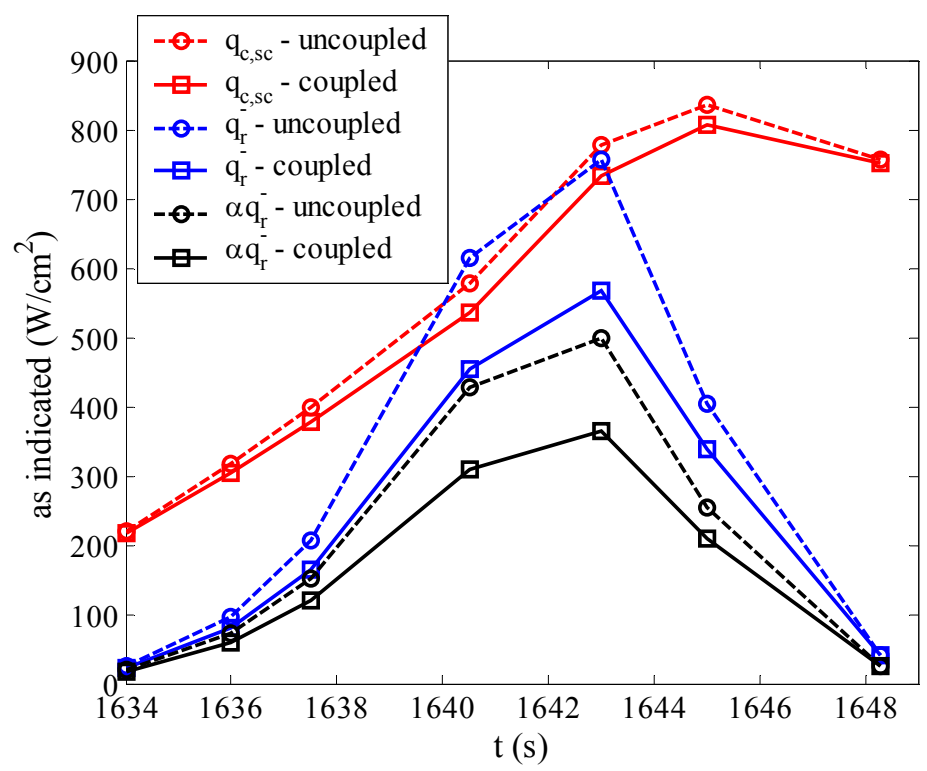

Figure 7. Uncoupled and coupled values for the convective and radiative heat flux along the Fire II trajectory. The $\alpha q_{r}$ values represent the radiative flux absorbed bv the calorimeter.
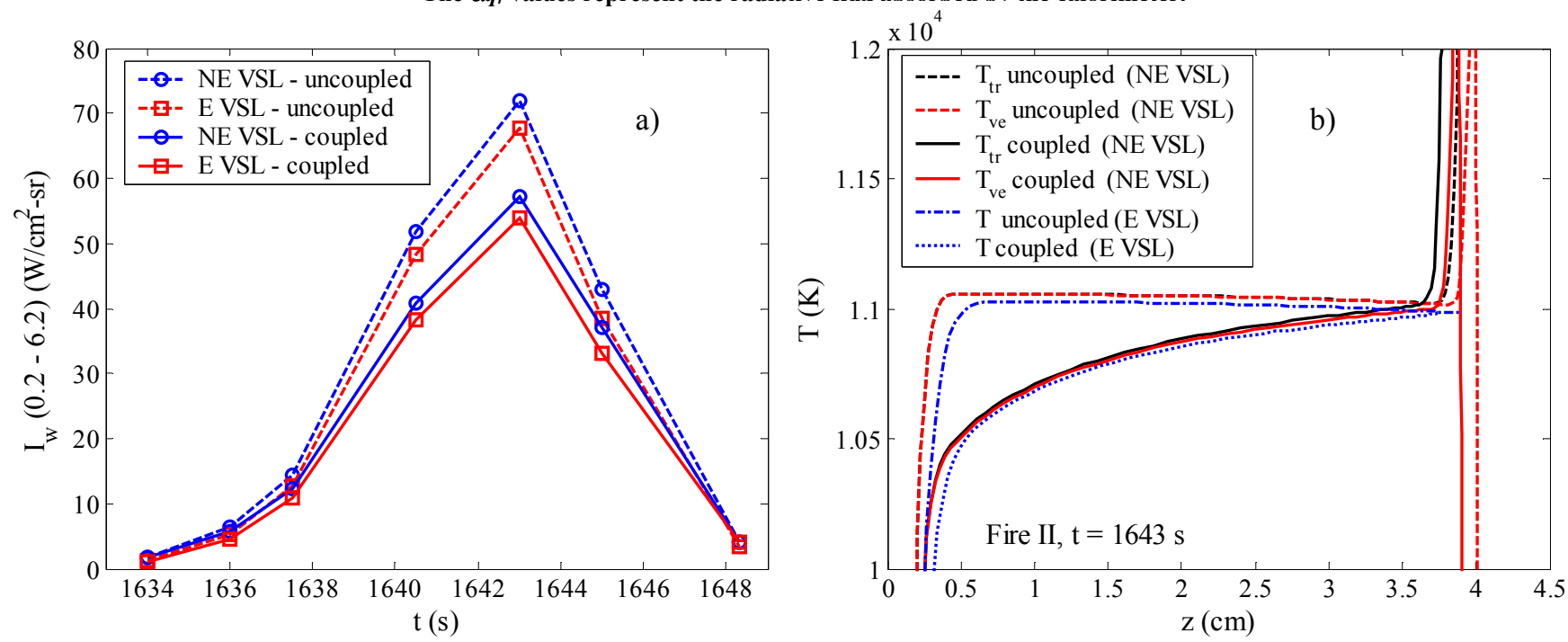

Figure 8. a) Partial wall intensity values $(0-6 \mathrm{eV})$ predicted by the coupled and uncoupled application of the NE and E VSL flowfield models. b) Uncoupled and coupled stagnation-line temperature profiles for $t=1643 \mathrm{~s}$.

To assess the influence of non-Boltzmann modeling and radiation-flowfield coupling on the radiative flux throughout the shock layer, and on the flux that ultimately reaches the wall, Figures 9 and 10 present the walldirected spectrally-integrated radiative flux profiles through the shock-layer and the radiative flux spectrum at the wall. In these figures, both the non-Boltzmann and Boltzmann results are presented for the coupled case, while only the non-Boltzmann result is presented for the uncoupled case. The non-Boltzmann and Boltzmann terminology used 
here specifies that the atomic and molecular electronic states are modeled by their respective Boltzmann or nonBoltzmann models. These models are discussed in detail in Johnston ${ }^{22}$. The accumulated flux values presented in Figures 9 and 10 allow for the contributions from the various radiative mechanisms to be compared (note that the spectrum for only the coupled non-Boltzmann case is presented in each figure). For the $1636 \mathrm{~s}$ case, the nonBoltzmann influence is seen to be large. This is a result of the relatively large region of nonequilibrium directly behind the shock, which was shown previously in Figure 5. From Figure 9, the increased flux for the Boltzmann case is seen to come from the atomic lines between 1.3 and $1.8 \mathrm{eV}$ and the $\mathrm{N}_{2}{ }^{+}$first-negative band system located between 2.0 and $5.0 \mathrm{eV}$. The atomic bound-free continuum located above $12.0 \mathrm{eV}$ also provides some excess radiation for the Boltzmann case. For the $1643 \mathrm{~s}$ case, Figure 10 shows that the Boltzmann influence is small. This is a result of most of the shock-layer being in equilibrium, which was shown by the temperature profiles presented in Figure 8(b). The influence of coupling for both the 1636 and $1643 \mathrm{~s}$ cases is seen to result mostly from the atomic lines located between 1.3 and $1.8 \mathrm{eV}$ and the atomic bound-free continuum located above $12.0 \mathrm{eV}$.
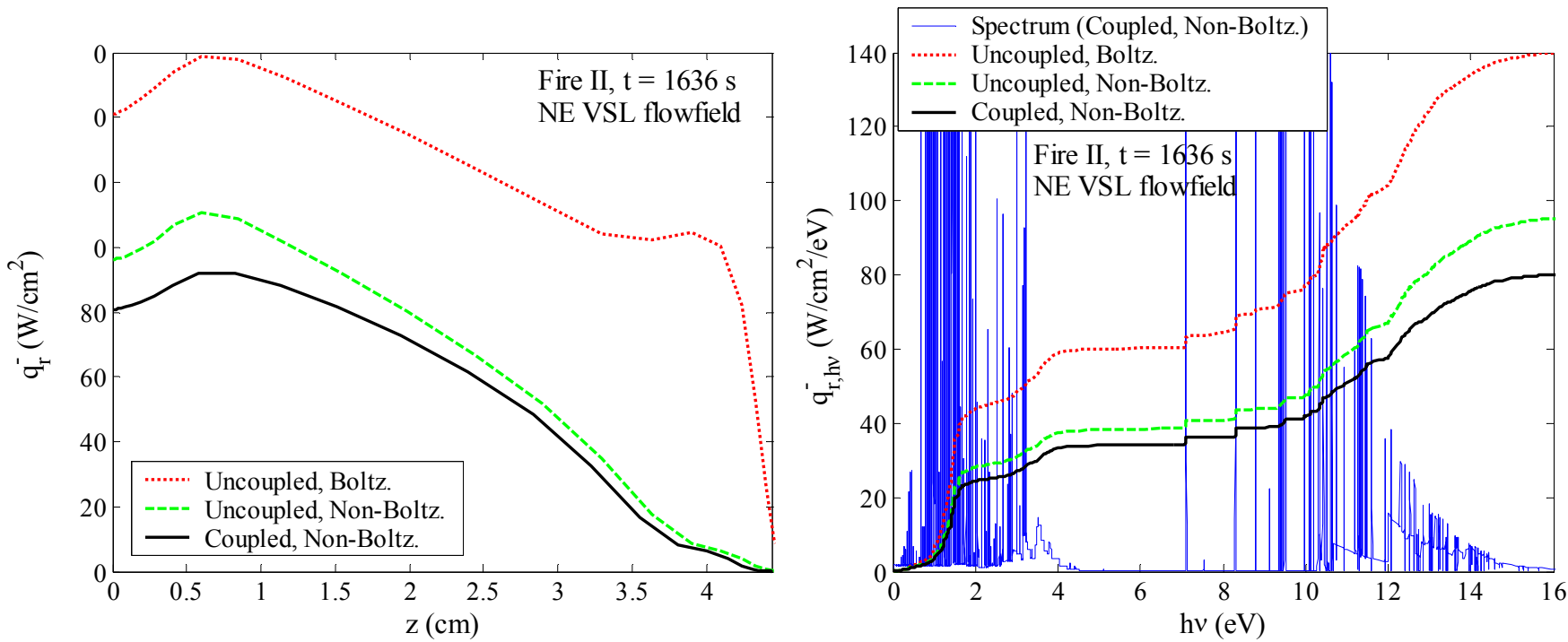

Figure 9. Wall-directed radiative flux profiles and wall spectra resulting from the coupled and uncoupled cases for $t=1636 \mathrm{~s}$.
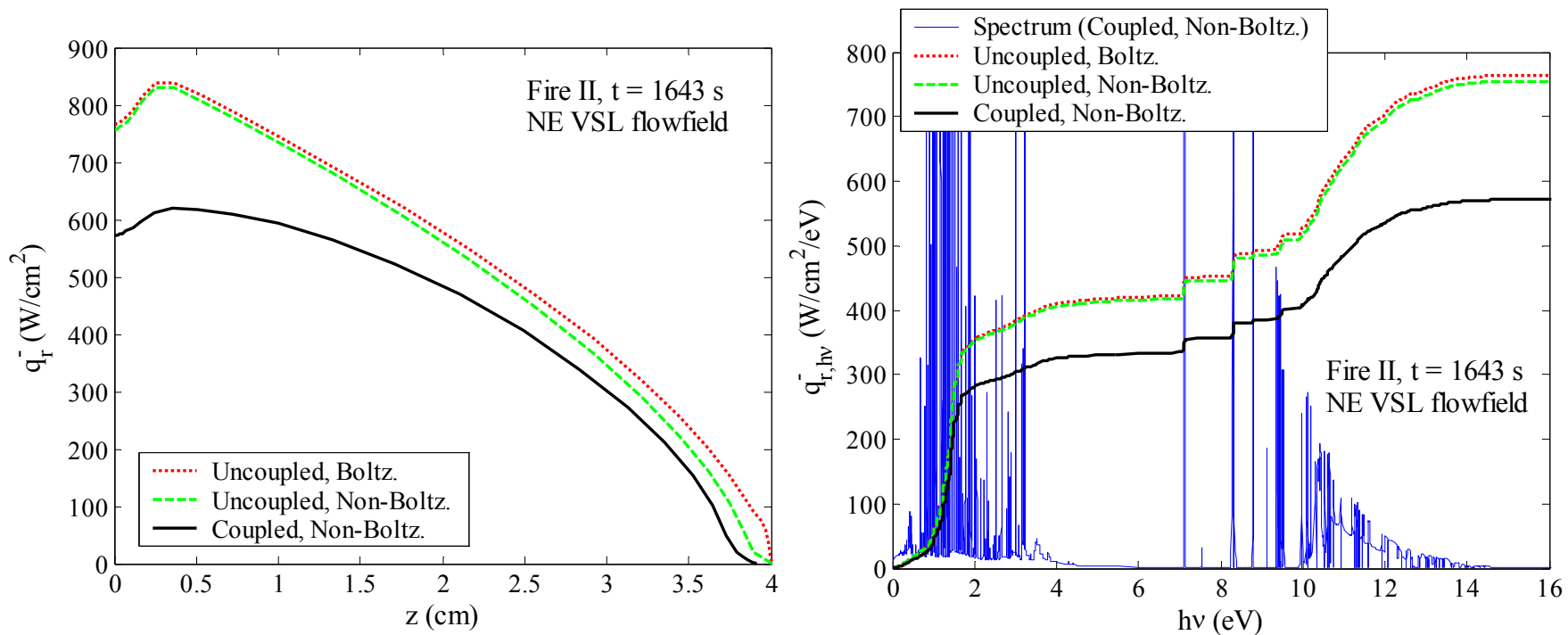

Figure 10. Wall-directed radiative flux profiles and wall spectra resulting from the coupled and uncoupled cases for $t=1643 \mathrm{~s}$.

The Fire II total radiometer data and the theoretical predictions of this data by past studies were presented previously in Section II. This data is compared in Figure 11 with the present radiation model coupled to the nonequilibrium (NE VSL) and equilibrium flowfield (E VSL) models. The predictions from past studies are also shown in these figures for comparison. Figure 11(b) shows the values for all trajectory points considered, while 
Figure 11(a) focuses on the three earliest points, which are important because they contain a significant amount of nonequilibrium radiation. From Figure 11(b) the differences between the flight data, previous predictions, and the present predictions can clearly be seen for the $1643 \mathrm{~s}$ point. For this trajectory point, the study by Olynick et al. ${ }^{12}$ is the only previous prediction that is closer to the data than the present NE VSL result. As discussed previously in this section, the Olynick et al. values are likely larger because of the reduced influence of radiation-flowfield coupling, resulting from the different treatment of the energy equations and temperatures. The Boltzmann assumption applied by Olynick et al. should not influence the radiation at $1643 \mathrm{~s}$ because the flowfield is mostly in equilibrium, as indicated in Figure 10. Note that the present equilibrium values (E VSL) are slightly larger than the values predicted by Gupta $^{6}$, who also applied an equilibrium VSL method. Assuming that the flowfield models were nearly equivalent, this trend is consistent with the fact that the present radiation model predicts larger radiation values for a given condition than the RAD/EQUIL code applied by Gupta. Also, the equivalent nose radius applied by Gupta (and Sutton) is $18 \%$ smaller than the currently applied radius, which would have possibly reduced the shockstandoff distances, and therefore the radiation values, below those predicted by the present model. Gupta does not present his predicted shock-standoff values, so this cannot be confirmed. The reasonable agreement with Park's ${ }^{13}$ prediction at $1643 \mathrm{~s}$ is noted. It should also be noted that the under-prediction at the 1637.5 point seen in Figure 11(a) is possibly a result of the radiometer window reaching its melting point, which would have caused the data at this trajectory point to be inaccurate. Of all the flight data values shown in Figure 11, this is the only point that was not considered a "prime" data period by Cauchon". For the early nonequilibrium points presented in Figure 11(a), the present results provide the most consistent agreement with the data, excluding the results of Olynick et al. ${ }^{12}$. The assumption made by Olynick et al. of a Boltzmann distribution of the electronic state populations should have caused a significant over-prediction of the radiation at the early trajectory points, as indicated by the behavior shown in Figure 9. Since this behavior was not predicted by Olynick et al., it may be concluded that their flowfield model was inconsistent with the present nonequilibrium VSL method, as well as past and present results from the LAURA Navier-Stokes code. A surprising result of Figure 11(a) is the relatively close agreement between the NE VSL and E VSL models, even though the shock layer is in considerable chemical nonequilibrium. This is a result of the nonBoltzmann model suppressing the radiation from the regions of chemical nonequilibrium in the NE VSL flowfield, as shown in Figure 9. Note that if the comparison between the NE and E VSL flowfields was made using a Boltzmann radiation model, the NE VSL prediction would be significantly larger than the E VSL prediction. Hence, if nonequilibrium chemistry is accounted for, a non-Boltzmann model must be applied.
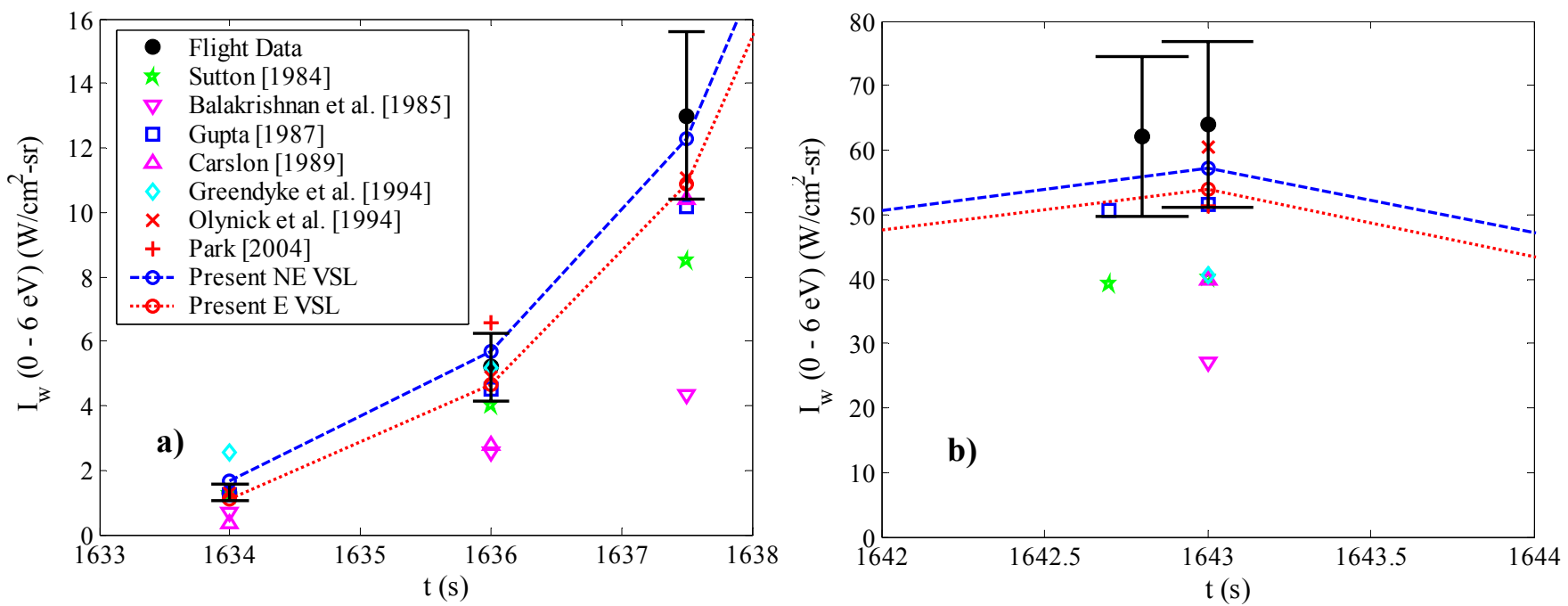

Figure 11. Comparison of the measured partial intensity $(0-6 \mathrm{eV})$ with various predictions, including the results of the present radiation model applied to the NE and E VSL codes: a) Early nonequilibrium trajectory points; b) Peak heating trajectory points.

The Fire II spectral radiometer data, which measured the intensity in the $2.2-4.1 \mathrm{eV}$ spectral range, was presented and compared with past predictions in Section II. The results of the present radiation model, coupled to the nonequilibrium (NE VSL) and equilibrium flowfield (E VSL) models, are compared with this data in Figure 12. The flight data is represented by two lines, representing the upper and lower limit of the data, which contained significant scatter. Good agreement is seen for the NE VSL method throughout the entire trajectory, while the E 
VSL results are slightly lower than the data. The radiation contained in this limited spectral range is due mostly to the $\mathrm{N}_{2}{ }^{+}$first negative band system. For the 1634 to 1640 s points, this radiation is controlled significantly by the rates chosen by Johnston ${ }^{22}$ for the excitation of the $\mathrm{N}_{2}{ }^{+}$(B) state. The agreement of the present model with the data at these points provides confidence that appropriate rates were chosen. Note that both Sutton's and Gupta's values are larger than the present predictions for the intensity from this $2.2-4.1 \mathrm{eV}$ spectral range. Since Figure 11(b) shows that the contribution from the $0-6 \mathrm{eV}$ range in these studies is actually smaller than the present predictions, then the radiation from the $0-2.2 \mathrm{eV}$ range must have been significantly under-predicted by Sutton and Gupta relative to the present model. This is likely a result of the increased line radiation in the $1-2 \mathrm{eV}$ range resulting from the updated set of the atomic lines applied in the present model, and discussed by Johnston ${ }^{22}$.

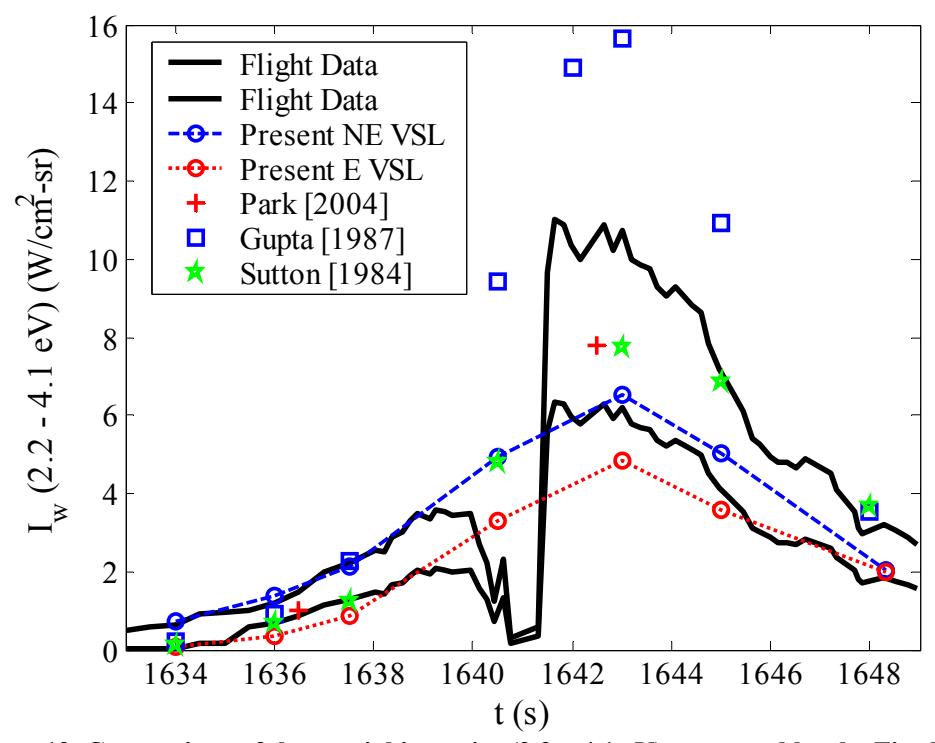

Figure 12. Comparison of the partial intensity $(2.2-4.1 \mathrm{eV})$ measured by the Fire II spectral radiometer with the present coupled results and previous predictions.

The Fire II total calorimeter measurements presented by Cornette ${ }^{3}$ contain the convective heating component along with the radiation absorbed by the beryllium calorimeter. This measured quantity, which will be labeled $q_{\text {total }}$, may be written as

$$
q_{\text {total }}=q_{c}+\alpha q_{r}^{-}
$$

where $q_{c}$ is the convective heating and $\alpha q_{r}$ represents the radiation absorbed by the beryllium calorimeter. The absorbed radiation was obtained by multiplying the frequency-dependent radiative flux by the frequency-dependent absorptance (of polished beryllium) presented by Cauchon ${ }^{2}$. The two components of Eq. (1) are presented in Tables 3 and 4, with both the super-catalytic (sc) and non-catalytic (nc) values presented in each table. Figure 13 compares the super-catalytic and non-catalytic values for $q_{\text {total }}$ with the flight data. For both the coupled and uncoupled supercatalytic cases, considering the flux from the $0-18 \mathrm{eV}$, a consistent over-prediction of flight data is seen to exist at all trajectory points except the last two. Interestingly, the coupled non-catalytic case, considering the flux from the 0 $-18 \mathrm{eV}$, is seen to compare well for all trajectory points, except the $1645 \mathrm{~s}$ point. This comparison supports the argument that the beryllium surface of the calorimeter, at temperatures ranging from 600 to $1,600 \mathrm{~K}$, is closer to being non-catalytic than super-catalytic. Note that, as indicated by Figure 3, the poor comparison at $1645 \mathrm{~s}$ is consistent with all previous theoretical predictions, and has not been explained. Although, due to the radiometer window reaching its melting point at this trajectory point, the radiometer measurement is not accurate enough to confirm the radiation prediction between 0 and $6 \mathrm{eV}$. Therefore, it is possible that some unknown radiation mechanism, not predicted by any previous study, contributed to the apparent shift of the peak heating point. Note that Sutton ${ }^{4}$ showed that this shift was not due to the values used for the beryllium absorptance.

Due to the strong absorption of VUV radiation and the limitations of instrumentation windows to transmit in this spectral region, it is difficult to obtain quality data to assess the accuracy of theoretical predictions for VUV radiation. The Fire II calorimeter provides the best flight experimental data to infer the magnitude of VUV radiation, although it requires an assumption that the predictions for the convective heating and the radiation in the 0 to $6 \mathrm{eV}$ spectral range are reasonably accurate. To indicate the influence of the vacuum ultraviolet (VUV) radiation in the 
present calculations, Figure 13 presents the super-catalytic convective heating plus the absorbed radiative heating between 0 and $6 \mathrm{eV}$ for both the coupled and uncoupled cases. The values presented here were taken from Tables 3 and 4, which means that the coupled cases considered the entire 0 to $18 \mathrm{eV}$ radiation contribution while calculating the flowfield (even for the cases where only the $0-6 \mathrm{eV}$ contribution is assumed absorbed by the calorimeter). It is seen that both the uncoupled and coupled values are below the flight data at the peak heating points.

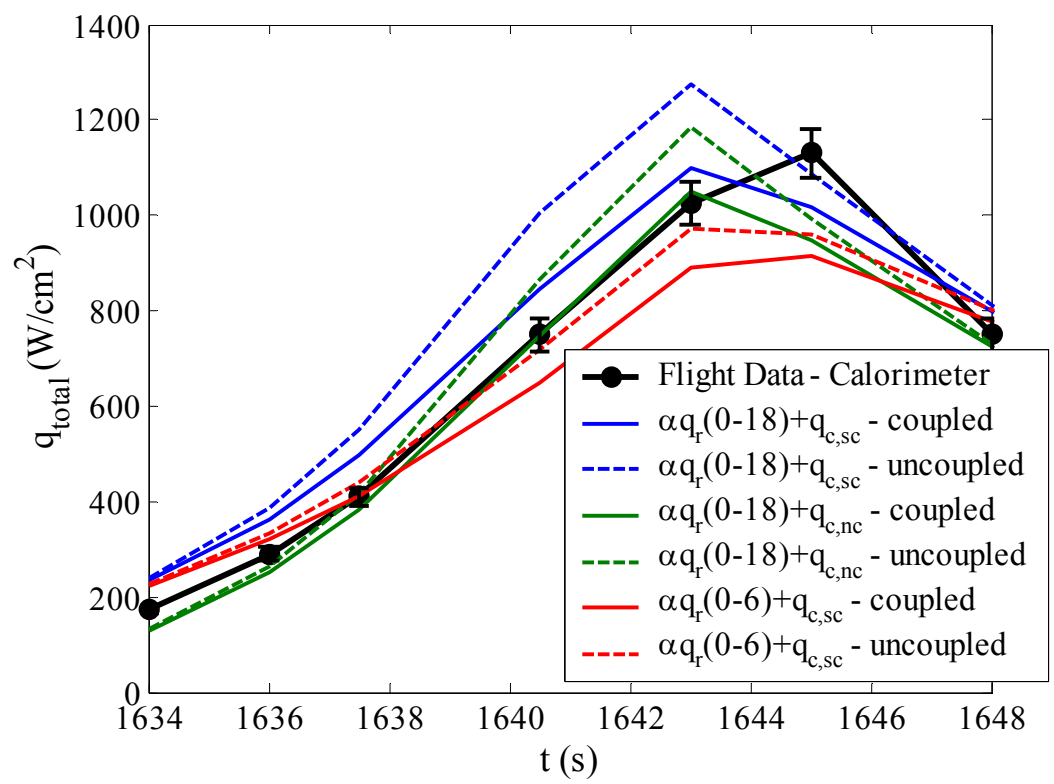

Figure 13. Comparison of the present results, assuming a catalytic and non-catalytic wall, with the Fire II calorimeter data. These are coupled values from the nonequilibrium VSL flowfield.

While Figure 13 implied the importance of the VUV radiation component by showing that it was required to achieve a good comparison between the calorimeter data and predictions, a stronger conclusion may be made regarding the VUV radiation if the comparison between the radiometer data and predictions are included in the discussion. Table 5 achieves this by converting the intensity (between 0 and $6 \mathrm{eV}$ ) measured by radiometer $\left(I_{w, \text { meas }}(0-6)\right)$ to the radiative flux absorbed by the calorimeter between 0 and $6 \mathrm{eV}\left(\alpha q_{r, \text { inferred }}(0-6)\right)$, which is then subtracted along with the predicted convective heating from the actual calorimeter measurement $\boldsymbol{q}_{\text {total,meas }}$, resulting in the radiative flux absorbed by the calorimeter between 6 and $18 \mathrm{eV}\left(\alpha q_{r \text {,inferred }}^{-}(6-18)\right)$. The subscript "inferred" is used to indicate that the value is a result of the actual calorimeter and radiometer data, along with the predicted convective heating and the ratio between the predicted $\alpha q_{r}^{-}(0-6)$ and $I_{w}(0-6)$ values, labeled $\beta$ in the table. Note that the predicted magnitude of the VUV radiation enters this analysis only indirectly through its influence on the coupled-radiation flowfield used to calculate $q_{c}, \alpha q_{r}$, inferred $(0-6)$, and $I_{w}(0-6)$. This makes the comparison between the "inferred" and predicted values in the last two columns of Table 5 meaningful. It is seen that, except for the 1643 and $1645 \mathrm{~s}$ points, the predicted values of the absorbed VUV radiation $\left(\alpha q_{r}^{-}(6-18)\right)$ fall between the $\alpha q_{r, \text { inferred }}^{-}(6-18)$ values obtained using the super-catalytic (not in parenthesis) and non-catalytic (in parenthesis) $q_{c}$ values. The negative $\alpha q_{r, \text { inferred }}^{-}(0-18)$ and $\alpha q_{r, \text { inferred }}^{-}(6-18)$ values in this table are a result of the $q_{\text {total,meas }}$ being lower than the predicted super-catalytic convective heating. The non-physical nature of these negative values suggest that the heatshield for this case is more accurately modeled with the non-catalytic assumption, which are shown in Table 5 to result in positive $\alpha q_{r \text {,inferred }}^{-}(0-18)$ and $\alpha q_{r \text {,inferred }}^{-}(6-18)$ values throughout the trajectory. The disagreement at $1645 \mathrm{~s}$ between the predicted and inferred values is not surprising, since it was not considered a "prime" data point by Cauchon $^{2}$ (although 1637.5 and 1640.5 were not either). On the other hand, the over-prediction of $\alpha q_{r}^{-}(6-18)$ relative to $\alpha q_{r \text {,inferred }}^{-}(6-18)$ (both the super-catalytic and non-catalytic value) at $1643 \mathrm{~s}$ is concerning because it is both a prime data point and the peak radiative heating point. A possible reason for this over-prediction is that the tangentslab approximation, applied for the radiation transport calculation, does not account for the shock-layer curvature. Past studies concerning the accuracy of the tangent slab approximation have concluded that a detailed threedimensional transport calculation produces a flux value about $10-20 \%$ lower than that predicted by the tangent slab approximation $^{38}$. Table 6 presents the values defined in Table 5, except now with both $\alpha q_{r}^{-}(0-6)$ and $\alpha q_{r}^{-}(6-18)$ multiplied by a factor of 0.85 . Only the values that are influenced by this factor are presented in Table 6 , with $\alpha q^{-}$ 
${ }_{r}(0-6), \alpha q_{r}^{-}(0-6)$, and $\alpha q_{r, \text { inferred }}^{-}(0-6)$ each being reduced by 0.85 while $\alpha q_{r, \text { inferred }}^{-}(6-18)$ is increased due to the reduction in $\alpha q_{r, \text { inferred }}^{-}(0-6)$. With this adjustment, the 1643 s prediction for $\alpha q_{r}^{-}(6-18)$ now falls between the supercatalytic and non-catalytic "inferred" values. Note that the intensity predictions do not require the tangent-slab approximation, and so the previous conclusions regarding the radiometer data are not influenced by this discussion.

Table 5. Manipulation of the Fire II radiometer and calorimeter data with predictions to isolate the absorbed flux measured by the calorimeter in the $6-18 \mathrm{eV}$ spectral range.

The columns are defined below the table.

\begin{tabular}{|c|c|c|c|c|c|c|c|c|c|}
\hline$t(\mathrm{~s})$ & $\begin{array}{c}I_{w, \text { meas }} \\
(\mathbf{0 - 6 )}\end{array}$ & $\beta$ & $\begin{array}{c}\alpha q_{r, \text { inferred }} \\
(\mathbf{0 - 6 )}\end{array}$ & $\begin{array}{c}\alpha q_{r}^{-} \\
(0-6)\end{array}$ & $q_{\text {total,meas }}$ & $\begin{array}{c}\alpha q_{r,}^{-} \text {inferred } \\
(\mathbf{0 - 1 8})\end{array}$ & $\begin{array}{c}\alpha q_{r}^{-} \\
(0-18)\end{array}$ & $\begin{array}{c}\alpha q_{r,}^{-} \text {inferred } \\
(\mathbf{6 - 1 8})\end{array}$ & $\begin{array}{c}\alpha q_{r}^{-} \\
(6-18)\end{array}$ \\
\hline 1634.0 & 1.3 & 3.1 & 4.1 & 5.3 & 175.0 & $-42.0(62.0)$ & 17.2 & $-46.1(57.9)$ & 11.9 \\
\hline 1636.0 & 5.2 & 2.9 & 15.2 & 16.7 & 290.0 & $-14.0(103)$ & 59.4 & $-29.2(87.8)$ & 42.7 \\
\hline 1637.5 & 13.0 & 2.8 & 37.0 & 35.0 & 410.0 & 33.0 (157) & 119 & $-4.0(120)$ & 83.9 \\
\hline 1640.5 & 35.0 & 2.7 & 96.1 & 112 & 750.0 & 215 (340) & 309 & $119(244)$ & 196 \\
\hline 1643.0 & 64.0 & 2.8 & 177.6 & 159 & 1025.0 & 292 (371) & 370 & $114(193)$ & 211 \\
\hline 1645.0 & 26.0 & 2.9 & 75.2 & 107 & 1130.0 & 323 (409) & 208 & $248(334)$ & 101 \\
\hline 1648.3 & 5.0 & 3.3 & 16.5 & 13.2 & 750.0 & $-2.0(78.0)$ & 25.6 & $-18.5(61.5)$ & 12.4 \\
\hline
\end{tabular}

$I_{w, \text { meas }}(0-6)=$ Intensity measured by the radiometer $\left(\mathrm{W} / \mathrm{cm}^{2} / \mathrm{sr}\right)$

$\beta=\alpha q_{r}{ }^{-}(0-6) / I_{w}(0-6)$, where both values are the predicted values.

$\alpha q_{r \text {, inferred }}(0-6)=\beta I_{w, \text { meas }}(0-6)$, equals the $\alpha q_{r}{ }^{-}(0-6)$ inferred by the radiometer measurement.

$\alpha q_{r}^{-}(0-6)=$ predicted value listed in Table 4.

$q_{\text {total,meas }}=$ Calorimeter measurement $=\alpha q_{r}{ }^{-}(0-18)+q_{c}$

$\alpha q_{r, \text { inferred }}(0-18)=q_{\text {total,meas }}-q_{c}$, where $q_{c}$ is the predicted value. The values not in parentheses use the super-catalytic $q_{c}$, while values in parentheses use the non catalytic $q_{c}$

$\alpha q_{r}^{-}(0-18)=$ predicted value listed in Table 4 .

$\alpha q_{r, \text { inferred }}^{-}(6-18)=\alpha q_{r}^{-}$, inferred $(0-18)-\alpha q_{r, \text { inferred }}^{-}(0-6)$. The values not in parentheses use the super-catalytic value for $\alpha q_{r,}^{-}$inferred $(0-18)$, while values in parentheses use the non-catalytic value.

$\alpha q_{r}^{-}(6-18)=$ predicted value listed in Table 4 .

Table 6. Comparison of the predicted and experimentally inferred values, defined in Table 5, but now accounting for the shock-wave curvature by multiplying $\beta, \alpha q_{r}^{-}(0-6)$ and $\alpha q_{r}^{-}(6-18)$ by 0.85 .

\begin{tabular}{|c|c|c|c|c|}
\hline$t(\mathrm{~s})$ & $\begin{array}{l}\alpha q_{r, \text { inferred }} \\
(\mathbf{0 - 6 )}\end{array}$ & $\begin{array}{c}\alpha q_{r}^{-} \\
(0-6)\end{array}$ & $\begin{array}{c}\alpha q_{r, \bar{n}} \text { inferred } \\
(\mathbf{6 - 1 8})\end{array}$ & $\begin{array}{c}\alpha q_{r}^{-} \\
(6-18)\end{array}$ \\
\hline 1634.0 & 3.4 & 4.5 & $-45.4(58.6)$ & 10.1 \\
\hline 1636.0 & 12.9 & 14.2 & $-26.9(90.0)$ & 36.3 \\
\hline 1637.5 & 31.4 & 29.8 & $-1.6(126)$ & 71.3 \\
\hline 1640.5 & 81.7 & 95.2 & $133(258)$ & 166.6 \\
\hline 1643.0 & 151.0 & 135.2 & $141(220)$ & 179.4 \\
\hline 1645.0 & 63.9 & 91.0 & 259 (345) & 85.8 \\
\hline 1648.3 & 14.0 & 11.2 & $-16.0(640)$ & 10.5 \\
\hline
\end{tabular}

\section{Conclusions}

The radiative heating for the Fire II flight experiment was studied using a radiation-flowfield coupled analysis consisting of a newly developed thermochemical nonequilibrium VSL analysis and a non-Boltzmann radiation model. Past studies of the Fire II flight data were shown to provide a relatively wide scatter of values, none of which compared well with the flight data in both the nonequilibrium and equilibrium regions of the trajectory. The present analysis was shown to provide a good comparison with the data in both of these trajectory regions. The updated atomic-line and non-Boltzmann models are the reason for this good comparison relative to previous models. The influence of chemical equilibrium and the Boltzmann assumption of the electronic state populations were studied. These were shown to have only a small influence on the radiative heating at peak heating conditions and a relatively large influence at the earlier nonequilibrium conditions. The influence of radiation-flowfield coupling was shown to reduce the radiative heating by about $30 \%$, while also slightly reducing the convective heating. An analysis of the calorimeter data indicated that the present model provides reasonable values for the vacuum ultraviolet radiation, which is shown to be a significant contributor to the total radiation. 


\section{References}

${ }^{1}$ Anderson, J.D., “An Engineering Survey of Radiating Shock Layers,” AIAA Journal, Vol. 7, pp. 1665-1675, 1969.

${ }^{2}$ Cauchon, D.L., "Radiative Heating Results from the Fire II Flight Experiment at a Reentry Velocity of 11.4 km/s," NASA TM X-1402, 1967.

${ }^{3}$ Cornette, E.S., "Forebody Temperature and Calorimeter Heating Rates Measured During Project Fire II Reentry At 11.35 $\mathrm{km} / \mathrm{s}$," NASA TM X-1305, 1966.

${ }^{4}$ Sutton, K., "Air Radiation Revisited," AIAA Paper 84-1733, 1984.

${ }^{5}$ Balakrishnan, A., Park, C., and Green, M., J., "Radiative Viscous-Shock-Layer Analysis of Fire, Apollo, and PAET Flight Data," AIAA Paper 85-1064, 1985.

${ }^{6}$ Gupta, R.N., "Navier-Stokes and Viscous Shock-Layer Solutions for Radiating Hypersonic Flows", AIAA Paper 87-1576, 1987.

${ }^{7}$ Bird, G.A., "Nonequilibrium Radiation During Re-Entry at 10 km/s," AIAA Paper 87-1543, 1987.

${ }^{8}$ Carlson, L.A., "Approximations for Hypervelocity Nonequilibrium Radiating, Reacting, and Conducting Stagnation Regions," Journal of Thermophysics and Heat Transfer, Vol. 3, pp. 380-388, 1989.

${ }^{9}$ Park, C., "Assessment of Two-Temperature Kinetic Model for Ionizing Air," Journal of Thermophysics and Heat Transfer, Vol. 3, pp. 233-244, July 1989,

${ }^{10}$ Gally, T.A., "Development of Engineering Methods for Nonequilibrium Radiative Phenomena about Aeroassisted Entry Vehicles, Ph.D. Dissertation, Texas A\&M University, 1992.

${ }^{11}$ Greendyke, R.B., and Hartung, L.C., "Convective and Radiative Heat Transfer Analysis for the Fire II Forebody," Journal of Spacecraft and Rockets, Vol. 31, pp. 986-992, 1994.

${ }^{12}$ Olynick, D.R., Henline, W.D., Chambers, L.H., and Candler, G.V., "Comparisons of Coupled Radiative Navier-Stokes Flow Solutions with the Project Fire II Flight Data," AIAA Paper 94-1955, 1994.

${ }^{13}$ Park, C., "Stagnation-Point Radiation for Apollo 4," Journal of Thermophysics and Heat Transfer, Vol. 18, pp. 349-357, 2004.

${ }^{14}$ Greendyke, R.B., and Hartung, L.C., "Approximate Method for the Calculation of Nonequilibrium Radiative Heat Transfer," Journal of Spacecraft and Rockets, Vol. 28, pp. 165-171, 1991.

${ }^{15}$ Hartung, L.C., Mitcheltree, R.A., Gnoffo, P.A., "Stagnation Point Nonequilibrium Radiative Heating and the Influence of Energy Exchange Models," Journal Thermophysics and Heat Transfer, Vol. 6, pp. 412-418, 1992.

${ }^{16}$ Park, C., "Problems of Rate Chemistry in the Flight Regimes of Aeroassisted Orbital Transfer Vehicles," AIAA 84-1730, 1984.

${ }^{17}$ Park, C., "Assessment of Two-Temperature Kinetic Model for Dissociating and Weakly Ionizing Nitrogen," Journal of Thermophysics and Heat Transfer, Vol. 2, pp. 8-16, July 1988.

${ }^{18}$ Park, C., "Assessment of Two-Temperature Kinetic Model for Ionizing Air," Journal of Thermophysics and Heat Transfer, Vol. 3, pp. 233-244, July 1989.

${ }^{19}$ Cauchon, D.L., Mckee, C.W., Cornette, E.S., "Spectral Measurements of Gas-Cap Radiation During Project Fire Flight Experiment at Reentry Velocities Near 11.4 km/s," NASA TM X-1389, 1967.

${ }^{20}$ Hartung, L.C., "Development of a Nonequilibrium Radiative Heating Prediction Method for Coupled Flowfield Solutions," Journal Thermophysics and Heat Transfer, Vol. 6, pp. 618-625, 1992.

${ }^{21}$ Greendyke, R.B., "Parametric Analysis of Radiative Structure in Aerobrake Shock Layers," Journal of Spacecraft and Rockets, Vol. 30, pp. 51-57, 1993.

22 Johnston, C. O., "Nonequilibrium Shock-Layer Radiative Heating for Earth and Titan Entry" Ph.D. Dissertation, Virginia Tech, November 2006.

${ }^{23}$ Davis, R.T., "Numerical Solution of the Hypersonic Viscous Shock-Layer Equations," AIAA Journal, Vol. 8, pp. 843-851, 1970.

${ }^{24}$ Gnoffo, P. A., Gupta, R. N., Shinn, J. L., "Conservation Equations and Physical Models for Hypersonic Air Flows in Thermal and Chemical Nonequilibrium," NASA TP-2867, 1989.

${ }^{25}$ Park, C., "Review of Chemical-Kinetic Problems of Future NASA Missions, I: Earth Entries," Journal of Thermophysics and Heat Transfer, Vol. 7, pp. 385-398, 1993.

${ }^{26}$ Mcbride, B.J., Zehe, M.J., and Gordon, S., "NASA Glenn Coefficients for Calculating Thermodynamic Properties of Individual Species," NASA TP 2002-211556, Sept. 2002

${ }^{27}$ Gupta, R.N., Yos, J.M., Thompson, R.A., and Lee, K.P., “A Review of Reaction Rates and Thermodynamic and Transport Properties for an 11-Species Air Model for Chemical and Thermal Nonequilibrium Calculations to 30,000 K", NASA RP-1232, 1990.

${ }^{28}$ Sutton, K., and Gnoffo, P.A., "Multi-Component Diffusion with Application to Computational Aerothermodynamics," AIAA Paper 98-2575, 1998.

${ }^{29}$ Ralchenko, Yu, et al., "NIST Atomic Spectra Database, Version 3.1.0," National Institute of Standards and Technology (NIST) Physics Lab, http://physics.nist.gov/PhysRefData/ASD/index. html, July 2006 [cited 3 Sept. 2006].

${ }^{30}$ Cunto, W., et al., "TOPbase at the CDS", Astronomy and Astrophysics, Vol. 275, pp. L5-L8, 1993 (The TOPbase website is http://vizier.u-strasbg.fr/topbase/topbase.html).

${ }^{31}$ Chambers, L.H., "Predicting Radiative Heat Transfer in Thermochemical Nonequilibrium Flow Fields," NASA TM-4564, 1994. 
${ }^{32}$ Laux, C. O., "Optical Diagnostics and Radiative Emission of Air Plasmas," High Temperature Gas Dynamics Lab, Mechanical Engineering Dept., Rept. T-288, Stanford University, 1993.

${ }^{33}$ Whang, T.J., Guoxing, Z., Stwalley, W.C., and Wu, C.Y.R., "Franck-Condon Factors of the ..... Transitions of $\mathrm{N}_{2}$," Journal of Quantitative Spectroscopy and Radiative Transfer, Vol. 55, 1996, pp. 335-344.

${ }^{34}$ Stahel, D., Leoni, M., and Dresslar, K., "Nonadiabatic Representations of the ${ }^{1} \Sigma_{\mathrm{u}}$ and ${ }^{1} \Pi_{\mathrm{u}}$ States of the $\mathrm{N}_{2}$ Molecule," Journal of Chemical Physics, Vol. 79, Sept 1983, pp. 2541-2558.

${ }^{35}$ Chauveau, S., Perrin, M.Y., Riviere, P., and Soufiani, A., "Contributions of Diatomic Molecular Electronic Systems to Heated Air Radiation," Journal of Quantitative Spectroscopy and Radiative Transfer, Vol. 72, 2002, pp. 503-530.

${ }^{36}$ Park, C., "Radiation Enhancement by Nonequilibrium in Earth's Atmosphere," Journal of Spacecraft and Rockets, Vol. 22, pp. 27-36, 1985.

${ }^{37}$ Hash, D., Olejniczak, J., Wright, M., Prabhu, D., Pulsonetti, M., Hollis, B., Gnoffo, P., Barhardt, M., Nompelis, I., and Candler, G., "Fire II Calculations for Hypersonic Nonequilibrium Aerothermodynamics Code Verification: DPLR, LAURA, and US3D," AIAA Paper 2007-605, January 2007.

${ }^{38}$ Hartung, L.C., and Hassan, H.A., "Radiation Transport Around Axisymmetric Blunt Body Vehicles Using a Modified Differential Approximation," Journal of Thermophysics and Heat Transfer, Vol. 7, 1993. 